

\title{
Global reliability-oriented sensitivity analysis under distribution parameter uncertainty
}

Vincent Chabridon, Mathieu Balesdent, Guillaume Perrin, Jérôme Morio, Jean-Marc Bourinet, Nicolas Gayton

\section{- To cite this version:}

Vincent Chabridon, Mathieu Balesdent, Guillaume Perrin, Jérôme Morio, Jean-Marc Bourinet, et al. Global reliability-oriented sensitivity analysis under distribution parameter uncertainty. 2020. hal-02877484

\section{HAL Id: hal-02877484 \\ https://hal.science/hal-02877484}

Preprint submitted on 22 Jun 2020

HAL is a multi-disciplinary open access archive for the deposit and dissemination of scientific research documents, whether they are published or not. The documents may come from teaching and research institutions in France or abroad, or from public or private research centers.
L'archive ouverte pluridisciplinaire HAL, est destinée au dépôt et à la diffusion de documents scientifiques de niveau recherche, publiés ou non, émanant des établissements d'enseignement et de recherche français ou étrangers, des laboratoires publics ou privés. 


\title{
Global reliability-oriented sensitivity analysis under distribution parameter uncertainty
}

\author{
Vincent Chabridon $^{\mathrm{a}, \mathrm{f}}$, Mathieu Balesdent ${ }^{\mathrm{b}}$, Guillaume Perrin ${ }^{\mathrm{c}}$, Jérôme \\ Morio $^{\mathrm{d}}$, Jean-Marc Bourinet ${ }^{\mathrm{e}}$, Nicolas Gayton ${ }^{\mathrm{e}}$ \\ ${ }^{a}$ EDF REDD, Département PRISME, 6 Quai Watier, 78401 Chatou, France \\ ${ }^{b}$ ONERA/DTIS, Université Paris Saclay, F-91123 Palaiseau Cedex, France \\ ${ }^{c} C E A / D A M / D I F, 91297$ Arpajon, France \\ ${ }^{d}$ ONERA/DTIS, Université de Toulouse, F-31055 Toulouse, France \\ ${ }^{e}$ SIGMA Clermont, F-63000 Clermont-Ferrand, France \\ ${ }^{f}$ Corresponding Author
}

\section{Introduction}

The design and analysis of complex engineering systems that one can find in major technical disciplines and industries (e.g., aerospace systems, nuclear power plants, bridges) rely intensely on both modeling and simulation. Modeling often implies to start from mathematical and physical equations in order to build relevant computer models. As for simulation, it aims at providing relevant and efficient numerical tools so as to simulate the systems' behavior, especially when physical phenomena cannot be directly observed nor measured experimentally. The computer models commonly used are said to be deterministic, meaning that for two identical runs with the same input values, the same model output will be obtained twice (i.e., there is no intrinsic stochasticity within the computer model). Such models are typically encountered in industrial applications when dealing with ordinary or partial differential equations solvers (e.g., finite element simulations in structural analysis, finite volume schemes for computational fluid dynamics). As a result, these codes are often complex to run, potentially costly-to-evaluate and sometimes, can be inserted in computational workflows. For these reasons, they can be considered as black-box models, i.e., models that are just considered by the user as an input-output function. Assessing the reliability in such a context requires to take the various sources of uncertainties which might affect the behavior of the system into account. Uncertainties may occur in 
the initial conditions, in the physical properties of the system (i.e., variability of mechanical properties of materials due the manufacturing process), in some environmental variables (i.e., uncertain loadings due to stochastic wind profiles) or finally, in the modeling itself (e.g., imperfect mathematical models of the real underlying physical phenoma, limited numerical accuracy). In this chapter, one will consider a probabilistic framework for the input uncertainty modeling assuming sufficient information is available to construct a relevant input probabilistic model (i.e., through assigning a structure of random variable to uncertain input variables). Once the sources of uncertainties have been clearly identified, it seems relevant to characterize them following whether the analyst can, in near-term and regarding a reasonable budget, reduce them (or not) so as to improve the accuracy of the predictions. From an engineering point of view, one can distinguish between two types of uncertainties:

- aleatory uncertainty refers to natural randomness or any irreducible uncertainty in a given context regarding a given maximum allowable budget;

- epistemic uncertainty refers to the lack of knowledge of the analyst and is potentially reducible by acquiring more information (i.e., data, measurements, expert judgements);

Following [1], such a distinction between aleatory and epistemic uncertainty should not be considered as a classification of phenomena, but only a pragmatic way of distinguishing between the uncertainties on which the analyst can allocate some budget for gaining knowledge and those for which it is impossible. Based on this classification, the analyst can take better informationbased choices. For the intested reader, more information about this topic can be found in Chapter 1.

In this chapter, one considers that aleatory uncertainty is taken into account through the use of an input probabilistic model, i.e., by assuming some probability distributions for the uncertain inputs (the type/shape of the law is supposed to be known). As for epistemic uncertainty, it is assumed to occur in the modeling of the input distributions (e.g., due to a lack of knowledge about the distribution parameters). This distribution parameter uncertainty potentially arises from statistical uncertainty (due to a limited amount of data) or could appear due to the absence of con- 
sensus in the expert elicitation process. As a result, one has to consider a bi-level input uncertainty composed of:

$\triangleright$ the aleatory uncertainty modeled by random variables;

$\triangleright$ the epistemic distribution parameter uncertainty.

Nonetheless, all these uncertainties have to be incorporated as soon as possible in the design process to ensure an optimized, reliable and safe design regarding standards and customers' requirements. This management of uncertainties can be achieved following a general uncertainty quantification methodology such as presented in Figure 1 [see, e.g., 2].

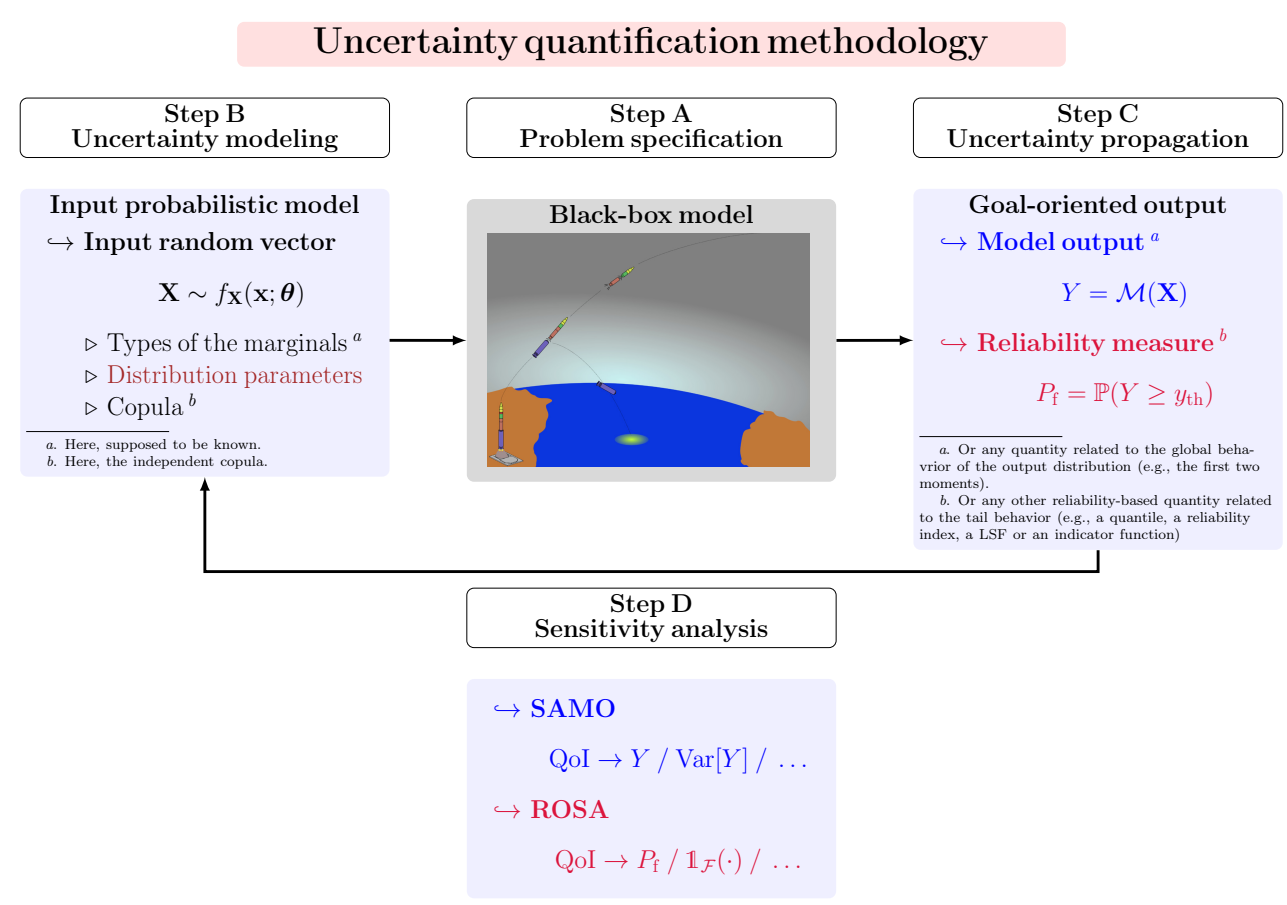

Figure 1: Illustration of the uncertainty quantification methodology.

Thus, starting from the black-box computer model and its specification (Step A), one has to choose a mathematical framework to model the input uncertainty (Step B). Then, the input uncertainties can be propagated 
through the computer model (Step C) so as to get the desired quantity of interest (QoI). This step is mainly driven by the choice of the QoI to estimate: for instance, one might be interested in the global characterization of the model output distribution (e.g., by computing its first two moments), or by the estimation of a high-order quantile (e.g., in the context of risk analysis), or finally by estimating an exceedance probability with respect to (w.r.t.) a safety threshold for reliability assessment. Such a probability is also denoted "failure probability" in the reliability community. Usually, in many industrial contexts, the sought failure probability is associated to a rare event, and is thus difficult to estimate. Once the QoI has been statistically estimated, one might be interested in performing a sensitivity analysis (SA) so as to investigate the robustness of the estimated QoI regarding the input uncertainty (Step D). As stressed by several authors, SA should be performed according to a range of general conceptual objectives, called "SA settings", properly defined in various references [see, e.g., 3]. As an example, one can mention the Factor Prioritization (FP-setting) which stipulates that the aim of SA could be to identify the key inputs driving the model behavior. Thus, a possible reduction of the uncertainty affecting these inputs might lead to the largest reduction of the output uncertainty. Another objective could be the Factor Fixing (FF-setting) which aims at identifying the noninfluential inputs which could be fixed at some given values without any loss of information about the output.

As already pointed out by several authors [see, e.g., 4, 5, 6], performing $\mathrm{SA}$ is subordinated to the choice of the QoI. Indeed, the key input variables responsible for the central part of the output distribution can be very different from the ones responsible for the tails (i.e., extreme quantiles). Thus, following the framework adopted in [7] ${ }^{1}$, one can consider that SA can be split into two types depending on the QoI being considered:

$\triangleright$ Sensitivity Analysis of Model Output (SAMO): gathering the set of methods for local, screening or global SA while considering the model output (e.g., focusing on the first moments or the entire

${ }^{1}$ Note that, in the paper from [8], the authors aim at developing a general approach for SA, named "Goal Oriented Sensitivity Analysis" (GOSA). The term "Reliability-Oriented Sensitivity Analysis" (ROSA) used in this work (not dedicated to a single method but to a wider class of methods) has been mainly borrowed and adapted from this reference. Note that the recent work of [9] adopted a similar semantics. 
range of the distribution) as the QoI. Comprehensive reviews about SAMO methods can be found in [10, 11];

$\triangleright$ Reliability-Oriented Sensitivity Analysis (ROSA): gathering the set of methods (from local to global ones) that constrain the SA to specific QoIs such as the typical reliability measures (e.g., failure probability, reliability index) or, in a broader sense, any restrictive QoI (e.g., binary indicator function of a failure event, values of the limit-state function, high- or low-order quantile, union of failure events). Such a definition is close to the notion of "target sensitivity analysis" recently proposed by [6]. More comprehensive reviews about ROSA methods can be found in [5] and [7].

To be more specific, the core differences between SAMO and ROSA can be briefly summarized as follows:

- the nature of the QoI: to make it simple, the idea is to not focus on the variability of the model output over its whole support (as done in SAMO), but on a more restrictive domain such as the left/right tail of the distribution [6];

- the problem of rare event probability estimation: the second difference is related to the fact that performing ROSA implies first to get "access" to the reliability measure. In the context of rare event probability estimation, this specific task can be difficult and/or computationally expensive to achieve and may require dedicated techniques. Futhermore, one needs to ensure the QoI has been accurately estimated so as to no introduce any strong bias in the ROSA. This part often implies to use properly tuned rare event probability estimation algorithms (see, e.g., Chapter 6 for more information about this topic);

- a reinterpretation of the traditional SA settings: as highlighted in [12] and [5], the specific context of ROSA implies to reinterpret the traditional SA settings listed previously. As an example, the FF-setting should be carefully examined as a realization of an undesired failure event might be due to a combination of several input effects, rather than a strong effect from a sole input.

For a broader and deeper review of existing ROSA methods, the interested reader is invited to refer to [5, 6] and [7]. 
In this chapter, one will mainly focus on a specific class of ROSA methods and its extension to reliability problems involving two uncertainty levels in input. This chapter is therefore organized as follows. Section 2 introduces the theoretical framework by introducing the core notations used in the following. Section 3 aims at presenting the Sobol indices on the indicator function. Section 4 proposes an extension of the previous indices to the case of a bilevel input uncertainty. Section 5 investigates an efficient strategy based on both simulation and kernel density estimation so as to get efficient estimators for the indices. Section 6 illustrates the benefits of such a methodology on different test-cases (two academic test-cases and one black-box computer code issued from aerospace research). Finally, a conclusion gathering the most important outcomes and some perspectives of this chapter are given in Section 7.

\section{Theoretical framework and notations}

The black-box computer model under study can be modeled as follows:

$$
\begin{array}{r|r}
\mathcal{M}: \mid \mathcal{D}_{\mathbf{X}} \subseteq \mathbb{R}^{d} & \longrightarrow \mathcal{D}_{Y} \subseteq \mathbb{R} \\
\mathbf{X}(\omega) & \longmapsto Y=\mathcal{M}(\mathbf{X}(\omega))
\end{array}
$$

where $\mathbf{X}=\left(X_{1}, \ldots, X_{d}\right)^{\top}$ is a $d$-dimensional second-order random vector gathering the input random variables. In the present study, these random variables are supposed to model the inputs affected by aleatory uncertainty (basically, the natural variability which is considered as irreducible). This continuous random vector is defined on a probability space $(\Omega, \mathcal{A}, \mathbb{P})$ with values in $\mathbb{R}^{d}$. In the rest of this chapter, the dependence over the sample space in $\mathbf{X}(\omega)$ will be omitted and the notation $\mathbf{x}$ will be used to denote a realization of the input random vector. One assumes that the joint probability density function $(\mathrm{PDF})$ of $\mathbf{X}$ exists and is given by $f_{\mathbf{X}}: \mathcal{D}_{\mathbf{X}} \subseteq \mathbb{R}^{d} \rightarrow \mathbb{R}_{+}$. This joint PDF is basically made of the marginal distributions (denoted by $f_{X_{i}}, i \in$ $\{1, \ldots, d\}$ ) and a stochastic dependence structure (i.e., a copula) between all these marginals. In this chapter, one assumes to be in the independent copula case $[13,14]$, meaning that the input variables are independent and, consequently, that the joint PDF is given by the product of the marginals. Moreover, it is assumed that the joint PDF belongs to a parametric family $\mathcal{P}_{\boldsymbol{\theta}}$ such that:

$$
\mathcal{P}_{\boldsymbol{\theta}}=\left\{f_{\mathbf{X}}(\cdot ; \boldsymbol{\theta}) \mid \boldsymbol{\theta} \in \mathcal{D}_{\boldsymbol{\theta}} \subseteq \mathbb{R}^{n_{\boldsymbol{\theta}}}\right\}
$$


where $\boldsymbol{\theta}$ stands for the vector of distribution parameters. In the following, the parametric dependence on $\boldsymbol{\theta}$ will be explicitly mentioned when necessary. Or else, it will be ommitted for the sake of conciseness.

Due to the uncertainties in input and by propagating them through the model $\mathcal{M}(\cdot)$ (which is assumed to be deterministic), one obtains the model output, denoted by $Y$. This quantity is a random variable (assumed to be scalar in this chapter) and is characterized by its PDF $f_{Y}: \mathcal{D}_{Y} \subseteq \mathbb{R} \rightarrow$ $\mathbb{R}_{+}$. The performance of the system of interest, modeled by $\mathcal{M}(\cdot)$, can be measured by a deterministic scalar function $g: \mathbb{R}^{d} \rightarrow \mathbb{R}$ called the limitstate function (LSF). A standard formulation for the LSF in the context of reliability assessment is given by:

$$
g(\mathbf{X})=y_{\text {th }}-\mathcal{M}(\mathbf{X})=y_{\text {th }}-Y
$$

where $y_{\text {th }} \in \mathbb{R}$ is a characteristic threshold output value beyond the one the system is considered as in a failure state. For any realization $\mathbf{x}$ of the input random vector, one can distinguish between two canonical domains: the failure domain given by $\mathcal{F}=\{\mathbf{x} \mid g(\mathbf{x}) \leq 0\}$ and the safe domain given by $\mathcal{S}=\{\mathbf{x} \mid g(\mathbf{x})>0\}$. The zero values of the LSF represent an hypersurface in $\mathbb{R}^{d}$ called the limit-state surface (LSS) which is defined by $\mathcal{F}^{0}=\{\mathbf{x} \mid g(\mathbf{x})=0\}$. Thus, by definition, the LSS belongs to the failure domain.

Finally, a widely used QoI in reliability assessment is the failure probabil$i t y$, denoted by $P_{\mathrm{f}}$, and given by the following $d$-fold integral:

$$
\begin{aligned}
P_{\mathrm{f}}=\mathbb{P}\left(Y \geq y_{\mathrm{th}}\right) & =\mathbb{P}(g(\mathbf{X}) \leq 0) \\
& =\int_{\mathcal{F}} f_{\mathbf{X}}(\mathbf{x}) \mathrm{d} \mathbf{x}=\int_{\mathcal{D}_{\mathbf{X}}} \mathbb{1}_{\mathcal{F}}(\mathbf{x}) f_{\mathbf{X}}(\mathbf{x}) \mathrm{d} \mathbf{x}=\mathbb{E}_{f_{\mathbf{X}}}\left[\mathbb{1}_{\mathcal{F}}(\mathbf{X})\right]
\end{aligned}
$$

where $\mathrm{d} \mathbf{x}=\mathrm{d} x_{1} \ldots \mathrm{d} x_{d}$ and $\mathbb{1}_{\mathcal{F}}(\cdot)$ is the indicator function of the failure domain defined such that $\mathbb{1}_{\mathcal{F}}(\mathbf{x})=1$ if $\mathbf{x} \in \mathcal{F}$ and $\mathbb{1}_{\mathcal{F}}(\mathbf{x})=0$ otherwise. In the rest of this chapter, it will be assumed that the failure event is rare, i.e., that the failure probability $P_{\mathrm{f}}$ is small (e.g., $P_{\mathrm{f}} \ll \frac{1}{N}$ with $N$ the available simulation budget). In daily engineering practice, rare event failure probabilities often vary from $10^{-2}$ to $10^{-9}$ [2]. The latter order of magnitude may often characterize highly-safe complex engineering systems for which the safety requirements are drastic (e.g., aerospace systems, nuclear power plants or civil engineering structures). 
From a practical point of view, a failure probability as defined in Eq. (4) can be estimated using various techniques: one the one hand, using approximation-based techniques such as the first/second-order reliability methods (FORM/SORM) or any metamodel-based technique such as, e.g., Gaussian processes or kriging, support vector machines and polynomial chaos expansions (see Chapter 7 for a review of a few kriging-based adaptive strategies); on the other hand, sampling-based techniques such as crude Monte Carlo sampling (CMC), importance sampling (IS), subset sampling (SS) (see Chapter 6 and [15] for more comprehensive reviews). In the following of this chapter, the two algorithms that will be used are crude Monte Carlo (CMC) sampling and subset sampling (SS) ${ }^{2}$.

For both CMC and SS, one needs to consider a sample $\left\{\mathbf{X}^{(j)}\right\}_{j=1}^{N}$ of $N$ independent and identically distributed (i.i.d.) copies of the input random vector $\mathbf{X}$ drawn according to $f_{\mathbf{X}}$ (in the following, this sentence will be denoted by $\left.\left\{\mathbf{X}^{(j)}\right\}_{j=1}^{N} \stackrel{\text { i.i.d. }}{\sim} f_{\mathbf{X}}\right)$. Therefore, one will consider the following elite set $\mathcal{E}=\left\{\mathbf{X}^{(j)}, 1 \leq j \leq N_{\text {fail }} \mid \mathbb{1}_{\mathcal{F}}\left(\mathbf{X}^{(j)}\right)=1\right\}$, i.e., the $N_{\text {fail }}$ samples that lead to failure regarding the indicator function (or, in other words, the samples which belong to the failure domain $\mathcal{F}$ ). These elite points are distributed according to the theoretical conditional $\mathrm{PDF} f_{\mathbf{X} \mid \mathcal{F}}$, which is unknown in practice. As a remark, in the specific context of rare event probability estimation, this elite set only contains a few points as the failure domain might be difficult to attain.

Once all the core necessary ingredients have been presented to achieve reliability assessment (i.e., failure probability estimation), Section 3 aims at presenting a class of global variance-based sensitivity indices adapted to ROSA.

\section{Global variance-based reliability-oriented sensitivity indices}

Among several global ROSA indices, variance-based ROSA indices play a major role as they have both strong theoretical and numerical properties and, depending on the context, an easier interpretability feature compared to ther indices [5, 7]. Known limits of variance-based indices are often related to their estimation cost and their lack of ability to catch high-order relationships

\footnotetext{
${ }^{2}$ Note that the formulation of Subset Simulation used in this chapter is consistent with the one proposed by [16]. This technique is detailed in Chapter 6 .
} 
which are not based on the variance [17]. However, in the ROSA context, their use has not been widely investigated till very recent works as shown in the following subsections.

\subsection{Introducing the Sobol indices on the indicator function}

Following [18], one can notice the following link between failure probabilities and mathematical expectations:

$$
P_{\mathrm{f}}-P_{\mathrm{f} \mid X_{i}}=\mathbb{E}\left[\mathbb{1}_{\mathcal{F}}(\mathbf{X})\right]-\mathbb{E}\left[\mathbb{1}_{\mathcal{F}}(\mathbf{X}) \mid X_{i}\right]
$$

where $P_{\mathrm{f} \mid X_{i}}$ is the conditional failure probability when $X_{i}$ is fixed at its mean value. Then, by squaring and taking the mean w.r.t. $X_{i}$ :

$$
\begin{aligned}
\mathbb{E}_{f_{X_{i}}}\left[\left(P_{\mathrm{f}}-P_{\mathrm{f} \mid X_{i}}\right)^{2}\right] & =\mathbb{E}_{f_{X_{i}}}\left[\left(\mathbb{E}\left[\mathbb{1}_{\mathcal{F}}(\mathbf{X})\right]-\mathbb{E}\left[\mathbb{1}_{\mathcal{F}}(\mathbf{X}) \mid X_{i}\right]\right)^{2}\right] \\
& =\operatorname{Var}\left[\mathbb{E}\left[\mathbb{1}_{\mathcal{F}}(\mathbf{X}) \mid X_{i}\right]\right]
\end{aligned}
$$

Finally, by dividing the last equation by the total variance $\operatorname{Var}\left[\mathbb{1}_{\mathcal{F}}(\mathbf{X})\right]$, one gets the following set of Sobol indices on the indicator function (proposed independently by [18] and [5]):

$$
\begin{aligned}
S_{i}^{\mathbb{1}_{\mathcal{F}}} & =\frac{\operatorname{Var}\left[\mathbb{E}\left[\mathbb{1}_{\mathcal{F}}(\mathbf{X}) \mid X_{i}\right]\right]}{\operatorname{Var}\left[\mathbb{1}_{\mathcal{F}}(\mathbf{X})\right]} \\
S_{T_{i}}^{\mathbb{1}_{\mathcal{F}}} & =1-\frac{\operatorname{Var}\left[\mathbb{E}\left[\mathbb{1}_{\mathcal{F}}(\mathbf{X}) \mid \mathbf{X}^{-i}\right]\right]}{\operatorname{Var}\left[\mathbb{1}_{\mathcal{F}}(\mathbf{X})\right]}
\end{aligned}
$$

where $S_{i}^{\mathbb{1}} \mathcal{F}$ is the first-order and and $S_{T_{i}}^{\mathbb{1}_{\mathcal{F}}}$ the total index associated to the variable $X_{i}$. Therefore, $\operatorname{Var}\left[\mathbb{1}_{\mathcal{F}}(\mathbf{X})\right]=P_{\mathrm{f}}\left(1-P_{\mathrm{f}}\right)$ and $\mathbf{X}^{-i}$ stands for $\mathbf{X}$ without the $i$-th component $X_{i}$. Moreover, note that, similarly to traditional Sobol indices in the SAMO context, the total index can be rewritten as [3]:

$$
S_{T_{i}}^{\mathbb{1}_{\mathcal{F}}}=\frac{\mathbb{E}\left[\operatorname{Var}\left[\mathbb{1}_{\mathcal{F}}(\mathbf{X}) \mid \mathbf{X}^{-i}\right]\right]}{\operatorname{Var}\left[\mathbb{1}_{\mathcal{F}}(\mathbf{X})\right]}
$$

Advanced sampling-based estimation schemes for these Sobol indices have been investigated in [19]. However, these numerical schemes still require a lot of simulations so as to achieve convergence. Very recently, another efficient estimation scheme using subset sampling has been proposed by [9]. This work is detailed hereafter. In the following, one will denote by the generic name" $S^{\mathbb{1}_{\mathcal{F}}}$-indices" the set of two indices given in Eq. (8) and Eq. (9). 
3.2. Rewriting Sobol indices on the indicator function using Bayes' theorem

Following [9], it is proposed to rewrite the $S^{\mathbb{1}_{\mathcal{F}}}$-indices presented hereabove as in the following Proposition 1 . Note that slightly similar derivations have been proposed in [20] in the context of moment-independent reliabilityoriented sensitivity indices.

Proposition 1 (Perrin and Defaux, 2019). The first and total order $S^{\mathbb{1}_{\mathcal{F}}}$ indices associated to each input $X_{i}, \forall i \in\{1 \ldots, d\}$, can be rewritten as follows:

$$
\begin{aligned}
S_{i}^{\mathbb{1}_{\mathcal{F}}} & =\frac{P_{\mathrm{f}}}{1-P_{\mathrm{f}}} \operatorname{Var}\left[\frac{f_{X_{i} \mid \mathcal{F}}\left(X_{i}\right)}{f_{X_{i}}\left(X_{i}\right)}\right] \\
S_{T_{i}}^{\mathbb{1}_{\mathcal{F}}} & =1-\frac{P_{\mathrm{f}}}{1-P_{\mathrm{f}}} \operatorname{Var}\left[\frac{f_{\mathbf{X}^{-i} \mid \mathcal{F}}\left(\mathbf{X}^{-i}\right)}{f_{\mathbf{X}^{-i}}\left(\mathbf{X}^{-i}\right)}\right]
\end{aligned}
$$

where:

$$
\begin{aligned}
f_{\mathbf{X} \mid \mathcal{F}}(\mathbf{x}) & =\frac{\mathbb{1}_{\mathcal{F}}(\mathbf{x}) f_{\mathbf{X}}(\mathbf{x})}{P_{\mathrm{f}}} \\
f_{X_{i} \mid \mathcal{F}}\left(x_{i}\right) & =\int_{\mathcal{D}_{\mathbf{X}^{-i}}} f_{\mathbf{X} \mid \mathcal{F}}(\mathbf{x}) \prod_{\substack{j=1 \\
j \neq i}}^{d} \mathrm{~d} x_{j}, \quad \mathcal{D}_{\mathbf{X}^{-i}}=\underset{\substack{j=1 \\
j \neq i}}{\mathbf{X}} \mathcal{D}_{X_{j}} \\
f_{\mathbf{X}^{-i} \mid \mathcal{F}}\left(\mathbf{x}^{-i}\right) & =\int_{\mathcal{D}_{X_{i}}} f_{\mathbf{X} \mid \mathcal{F}}(\mathbf{x}) \mathrm{d} x_{i} .
\end{aligned}
$$

Proof. A sketch of proof can be found in [9]. However, more details are given below for the sake of clarity. Recalling that $\operatorname{Var}\left[\mathbb{1}_{\mathcal{F}}(\mathbf{X})\right]=P_{\mathrm{f}}\left(1-P_{\mathrm{f}}\right)$ and applying Bayes' theorem to Eq. (7), one gets:

$$
\begin{aligned}
\mathbb{E}_{f_{X_{i}}}\left[\left(P_{\mathrm{f}}-P_{\mathrm{f} \mid X_{i}}\right)^{2}\right] & =\mathbb{E}_{f_{X_{i}}}\left[\left(P_{\mathrm{f}}-P_{\mathrm{f}} \frac{f_{X_{i} \mid \mathcal{F}}\left(x_{i}\right)}{f_{X_{i}}\left(x_{i}\right)}\right)^{2}\right] \\
& =P_{\mathrm{f}}^{2} \mathbb{E}_{f_{X_{i}}}\left[\left(1-\frac{f_{X_{i} \mid \mathcal{F}}\left(x_{i}\right)}{f_{X_{i}}\left(x_{i}\right)}\right)^{2}\right] .
\end{aligned}
$$

Hence, for the first order index, by applying the total variance theorem, one 
gets:

$$
\begin{aligned}
S_{i}^{\mathbb{1}_{\mathcal{F}}} & =\frac{\mathbb{E}_{f_{X_{i}}}\left[\left(P_{\mathrm{f}}-P_{\mathrm{f} \mid X_{i}}\right)^{2}\right]}{P_{\mathrm{f}}\left(1-P_{\mathrm{f}}\right)} \\
& =\frac{P_{\mathrm{f}}}{1-P_{\mathrm{f}}}\left(\operatorname{Var}\left[1-\frac{f_{X_{i} \mid \mathcal{F}}\left(x_{i}\right)}{f_{X_{i}}\left(x_{i}\right)}\right]+\mathbb{E}_{f_{X_{i}}}\left[1-\frac{f_{X_{i} \mid \mathcal{F}}\left(x_{i}\right)}{f_{X_{i}}\left(x_{i}\right)}\right]^{2}\right) \\
& =\frac{P_{\mathrm{f}}}{1-P_{\mathrm{f}}}\left(\operatorname{Var}\left[\frac{f_{X_{i} \mid \mathcal{F}}\left(x_{i}\right)}{f_{X_{i}}\left(x_{i}\right)}\right]+1-\mathbb{E}_{f_{X_{i}}}\left[\frac{f_{X_{i} \mid \mathcal{F}}\left(x_{i}\right)}{f_{X_{i}}\left(x_{i}\right)}\right]^{2}\right) .
\end{aligned}
$$

Moreover, one can notice that:

$$
\begin{aligned}
\mathbb{E}_{f_{X_{i}}}\left[\frac{f_{X_{i} \mid \mathcal{F}}\left(x_{i}\right)}{f_{X_{i}}\left(x_{i}\right)}\right] & =\int_{\mathcal{D}_{X_{i}}} \frac{f_{X_{i} \mid \mathcal{F}}\left(x_{i}\right)}{f_{X_{i}}\left(x_{i}\right)} f_{X_{i}}\left(x_{i}\right) \mathrm{d} x_{i} \\
& =\int_{\mathcal{D}_{X_{i}}} \int_{\mathcal{D}_{\mathbf{X}^{-i}}} f_{\mathbf{X} \mid \mathcal{F}}(\mathbf{x}) \prod_{j=1}^{d} \mathrm{~d} x_{j} \mathrm{~d} x_{i} \\
& =\int_{\mathcal{D}_{\mathbf{X}}} \frac{1}{P_{\mathrm{f}}} \mathbb{1}_{\mathcal{F}}(\mathbf{x}) f_{\mathbf{X}}(\mathbf{x}) \mathrm{d} \mathbf{x} \\
& =1
\end{aligned}
$$

which finally allows to get the expected result. Similar derivations for the total index can be achieved starting from:

$$
\mathbb{E}_{f_{\mathbf{X}^{-i}}}\left[\left(P_{\mathrm{f}}-P_{\mathrm{f} \mid \mathbf{X}^{-i}}\right)^{2}\right]=P_{\mathrm{f}}^{2} \mathbb{E}_{f_{\mathbf{X}^{-i}}}\left[\left(1-\frac{f_{\mathbf{X}^{-i} \mid \mathcal{F}}\left(\mathbf{x}^{-i}\right)}{f_{\mathbf{X}^{-i}}\left(\mathbf{x}^{-i}\right)}\right)^{2}\right]
$$

and noticing that $\mathbb{E}_{f_{\mathbf{X}^{-i}}}\left[\frac{f_{\mathbf{X}^{-i} \mid \mathcal{F}}\left(\mathbf{x}^{-i}\right)}{f_{\mathbf{X}^{-i}}\left(\mathbf{x}^{-i}\right)}\right]=1$.

As a result, the two estimators associated to the first-order and total 
indices are given by:

$$
\begin{aligned}
& \widehat{S}_{i}{ }^{1}{ }_{\mathcal{F}}=\frac{\widehat{P}_{\mathrm{f}}}{1-\widehat{P}_{\mathrm{f}}} \operatorname{Var}\left[\frac{\widehat{f}_{X_{i} \mid \mathcal{F}}\left(X_{i}\right)}{f_{X_{i}}\left(X_{i}\right)}\right] \\
& \widehat{S}_{T_{i}}^{\mathbb{1}_{\mathcal{F}}}=1-\frac{\widehat{P}_{\mathrm{f}}}{1-\widehat{P}_{\mathrm{f}}} \operatorname{Var}\left[\frac{\widehat{f}_{\mathbf{X}^{-i} \mid \mathcal{F}}\left(\mathbf{X}^{-i}\right)}{f_{\mathbf{X}^{-i}}\left(\mathbf{X}^{-i}\right)}\right]
\end{aligned}
$$

where $\widehat{f}_{X_{i} \mid \mathcal{F}}$ is the estimator of the marginal PDF of $X_{i}$ at failure, while $\widehat{f}_{\mathbf{X}^{-i} \mid \mathcal{F}}$ is the estimator of the joint PDF of $\mathbf{X}^{-i}$ at failure, i.e., a $(d-1)$-dimensional PDF.

This set of estimators presents the main advantage of requiring only a post-processing phase of a single elite set $\mathcal{E}$ obtained from a reliability analysis using any sampling-based technique mentioned in Section 2. However, the difficulty remains the accurate estimation of the densities, especially for the total indices which involve the estimation of a multivariate density at failure.

In the following, one proposes to adapt the previous set of estimators and the efficient estimation strategy to the bi-level input uncertainty problem. This part consists in the core technical contribution of this chapter.

\section{Sobol indices on the indicator function adapted to the bi-level input uncertainty}

\subsection{Reliability analysis under distribution parameter uncertainty}

In this chapter, two levels of uncertainty are considered: the first one represents the variability in the basic input variables and thus affects the input random vector $\mathbf{X}$ when the second one represents the lack of knowledge affecting the distribution parameters. To do so, the following Bayesian hierarchical model [21] is considered:

$$
\begin{array}{rlrl}
\mathbf{X} & \sim f_{\mathbf{X} \mid \boldsymbol{\Theta}}(\mathbf{x} \mid \boldsymbol{\theta}): \mathcal{D}_{\mathbf{X}} \subseteq \mathbb{R}^{d} \rightarrow \mathbb{R}_{+} & \text {(uncertainty level \#1) } \\
\boldsymbol{\Theta} \sim f_{\boldsymbol{\Theta} \mid \boldsymbol{\xi}}(\boldsymbol{\theta} \mid \boldsymbol{\xi}): \mathcal{D}_{\boldsymbol{\Theta}} \subseteq \mathbb{R}^{n_{\boldsymbol{\theta}}} \rightarrow \mathbb{R}_{+} & \text {(uncertainty level \#2) } \\
\boldsymbol{\xi} & =\left(\xi_{1}, \xi_{2}, \ldots, \xi_{n_{\boldsymbol{\xi}}}\right)^{\top} \in \mathbb{R}^{n_{\boldsymbol{\xi}}} & & \text { (deterministic level). }
\end{array}
$$

In this hierarchical representation, one can distinguish three layers of inputs:

- the first layer is constituted by the random vector $\mathbf{X}$ gathering the basic stochastic variables. Based on prior knowledge, a probability distribution can be assumed through the choice of a parametric model; 
- the second layer is constituted by uncertain and deterministic (i.e., supposed to be known accurately enough) distribution parameters. Adding such a layer is consistent with the Bayesian point of view of modeling either "uncertain" (in the sense of stochastic) or "unknown but fixed" parameters. In the present chapter, the prior distribution is mostly assumed to be derived from expert judgment or from a limited set of data. Thus, despite the fact that Bayes' theorem is not used as an updating procedure, one can consider that this prior distribution characterizes the epistemic uncertainty affecting the distribution parameters;

- the third layer is composed of fixed hyper-parameters gathered in $\boldsymbol{\xi}$. These hyper-parameters, which can be either some moments or bounds, characterize the prior distributions of uncertain parameters $\Theta$.

The treatment of distribution parameter uncertainty in reliability assessment has been originally described in several seminal works such as [22, 23]. Then, extensions of the previous works have been proposed in $[24,21,1]$. Therefore, in such a context of bi-level input uncertainty, an interesting reliability measure can be the predictive failure probability, defined such that:

$$
\begin{aligned}
\widetilde{P}_{\mathrm{f}}(\boldsymbol{\xi}) \stackrel{\text { def }}{=} \mathbb{E}_{f_{\Theta \mid \xi}}\left[P_{\mathrm{f}}(\boldsymbol{\Theta})\right] & =\mathbb{E}_{f_{\Theta \mid \boldsymbol{\xi}}}\left[\mathbb{E}_{f_{\mathbf{X} \mid \Theta}}\left[\mathbb{1}_{\mathcal{F}}(\mathbf{X}) \mid \boldsymbol{\Theta}\right] \mid \boldsymbol{\xi}\right] \\
& =\int_{\mathcal{D}_{\Theta}} P_{\mathrm{f}}(\boldsymbol{\theta}) f_{\Theta \mid \boldsymbol{\xi}}(\boldsymbol{\theta} \mid \boldsymbol{\xi}) \mathrm{d} \boldsymbol{\theta}
\end{aligned}
$$

where $P_{\mathrm{f}}(\boldsymbol{\theta})$ is the conditional failure probability ${ }^{3}$ given by:

$$
\begin{aligned}
P_{\mathrm{f}}(\boldsymbol{\theta})=\mathbb{P}(g(\mathbf{X}) \leq 0 \mid \boldsymbol{\Theta}=\boldsymbol{\theta}) & =\int_{\mathcal{D}_{\mathbf{X}}} \mathbb{1}_{\mathcal{F}}(\mathbf{x}) f_{\mathbf{X} \mid \boldsymbol{\Theta}}(\mathbf{x} \mid \boldsymbol{\theta}) \mathrm{d} \mathbf{x} \\
& =\mathbb{E}_{f_{\mathbf{X} \mid \Theta}}\left[\mathbb{1}_{\mathcal{F}}(\mathbf{X}) \mid \boldsymbol{\Theta}=\boldsymbol{\theta}\right]
\end{aligned}
$$

As highlighted in [25], an efficient way to estimate such a QoI can be achieved by considering a so-called "augmented" random vector $\mathbf{Z} \stackrel{\text { def }}{=}(\mathbf{X}, \boldsymbol{\Theta})^{\top}$ defined on $\mathcal{D}_{\mathbf{Z}}=\mathcal{D}_{\mathbf{X}} \times \mathcal{D}_{\Theta}$ (where $\times$ is the Cartesian product) with joint PDF

\footnotetext{
${ }^{3}$ Please note that the conditional failure probability defined here differs from the one defined in Eq. (5).
} 
$f_{\mathbf{Z} \mid \boldsymbol{\xi}}(\mathbf{z} \mid \boldsymbol{\xi}) \stackrel{\text { def }}{=} f_{(\mathbf{X}, \boldsymbol{\Theta}) \mid \boldsymbol{\xi}}((\mathbf{x}, \boldsymbol{\theta}) \mid \boldsymbol{\xi})=f_{\mathbf{X} \mid \boldsymbol{\Theta}}(\mathbf{x} \mid \boldsymbol{\theta}) f_{\boldsymbol{\Theta} \mid \boldsymbol{\xi}}(\boldsymbol{\theta} \mid \boldsymbol{\xi})$ such that the expression in Eq. (21b) can be rewritten as follows:

$$
\begin{aligned}
\widetilde{P}_{\mathrm{f}}(\boldsymbol{\xi}) & =\int_{\mathcal{D}_{\Theta}} \int_{\mathcal{D}_{\mathbf{X}}} \mathbb{1}_{\mathcal{F}}(\mathbf{x}) f_{\mathbf{X} \mid \boldsymbol{\Theta}}(\mathbf{x} \mid \boldsymbol{\theta}) f_{\boldsymbol{\Theta} \mid \boldsymbol{\xi}}(\boldsymbol{\theta} \mid \boldsymbol{\xi}) \mathrm{d} \mathbf{x} \mathrm{d} \boldsymbol{\theta} \\
& =\int_{\mathcal{D}_{\mathbf{Z}}} \mathbb{1}_{\mathcal{F}_{\mathbf{z}}}(\mathbf{z}) f_{\mathbf{Z} \mid \boldsymbol{\xi}}(\mathbf{z} \mid \boldsymbol{\xi}) \mathrm{d} \mathbf{z} \\
& =\mathbb{E}_{f_{\mathbf{Z} \mid \boldsymbol{\xi}}}\left[\mathbb{1}_{\mathcal{F}_{\mathbf{Z}}}(\mathbf{Z}) \mid \boldsymbol{\xi}\right]
\end{aligned}
$$

where $\mathcal{F}_{\mathbf{z}}=\left\{\mathbf{z} \in \mathcal{D}_{\mathbf{z}} \mid g(\mathbf{z}) \leq 0\right\}$. This formulation, named "Augmented Reliability Approach" (ARA) numerically implies to estimate the expected value in Eq (23c). The coupling between the ARA framework to handle distribution parameter uncertainty and rare event probability estimation (e.g., ARA/CMC and ARA/SS) has been studied in [25].

In the following, one will only focus on the first two layers in input, i.e., $\mathbf{X}$ and $\boldsymbol{\Theta}$. Thus, for the sake of conciseness, the third layer (i.e., the hyper-parameters $\boldsymbol{\xi}$ ) will be omitted. The dependence on $\boldsymbol{\xi}$ is interesting in the particular case of failure probability robustess analysis using local ROSA such as proposed in [26].

\subsection{Bi-level input uncertainty: aggregated vs. disaggregated types of uncer- tainty}

When dealing with the bi-level input uncertainty as contained in the hierarchical model described previously, two cases can be considered:

- on the one hand, one might be interested in the combined effects of both aleatory and epistemic uncertainties at failure, i.e., one might want to "aggregate" both sources of uncertainty;

- on the other hand, one might be rather interested in distinguishing between the contributions of aleatory and epistemic uncertainties, i.e., one might want to "disaggregate" (or "separate") both sources of uncertainty.

Concerning the first problem, adapting the previous $S^{\mathbb{1}_{\mathcal{F}}}$-indices can be achieved by considering the equivalent indices evaluated on the augmented vector $\mathbf{Z}=(\mathbf{X}, \mathbf{\Theta})^{\top}$. Such indices would reflect the contribution of both the variables $\mathbf{X} \mid \Theta$ and distribution parameters $\Theta$. 
In the second problem, the idea is to study the impact of aleatory and epistemic uncertainties separately. Indeed, as stressed by several authors such as [27, 28], separating both contributions of aleatory and epistemic uncertainties may be of utmost importance if one desires to get a deeper insight about which type of uncertainty plays a major role on the system at failure (see Chapter 1). Moreover, it allows to build a well-informed decision process in terms of budget allocation to possibly reduce epistemic uncertainty if this one plays a significant role on the variability of the reliability measure.

In this chapter, it is proposed to consider a disaggregated version of the augmented input vector to separate both types of uncertainty. Moreover, the effects of both types of uncertainty on the indicator function (in other words, "at failure") are studied and taken into account in the ROSA context, as presented in the following sections.

\subsection{Disaggregated random variables}

One possible manner to study the effects of distribution parameter uncertainty and input variability separately is to transform the input variables as proposed in [29] (in the context of p-boxes). As a preliminary example, one can consider the Gaussian case as an illustrative example. Thus, under a single-level type of uncertainty, a Gaussian random variable $X$ can be split as follows:

$$
X=\mu_{X}+\sigma_{X} U_{X}
$$

where $\boldsymbol{\theta}=\left(\mu_{X}, \sigma_{X}\right)^{\top}$ are the distribution parameters and $U_{X} \sim \mathcal{N}(0,1)$ is a standard Gaussian variable which characterizes the inherent variability of $X$. Note that such a transformation is not limited to the Gaussian case. One can build similar transformations for random variables following, for instance, lognormal, Gumbel or Weibull distributions. The interested reader should refer to [29] for the presentation of these cases.

Under a bi-level input uncertainty, the idea is to consider a prior distribution $f_{\Theta}$ over the uncertain distribution parameters $\boldsymbol{\Theta}=\left(M_{X}, S_{X}\right)^{\top}$. As a consequence, the previous decomposition can be rewritten as follows:

$$
X=M_{X}+S_{X} U_{X}
$$

where $\mathbf{V}_{\text {dis }}=\left(M_{X}, S_{X}, U_{X}\right)^{\top}$ is a vector of independent variables denoted as the "disaggregated augmented vector". Note that, for some cases, only the 
mean value or the standard deviation could be considered as uncertain. In the following, the study will be limited to the Gaussian case as working in the u-space will facilitate the calculations, without loss of generality. Finally, the augmented input vector under a bi-level uncertainty will be composed of two sorts of inputs: the inputs that are not affected by the bi-level uncertainty (gathered in the vector $\mathbf{X}_{\text {single }}$ ) and the disaggregated ones, such that $\mathbf{Z}=$ $\left(\mathbf{V}_{\text {dis }}, \mathbf{X}_{\text {single }}\right)^{\top}$ with $d_{\mathbf{Z}}=d_{\text {dis }}+d_{\text {single }}$ denoting the dimension of this vector. As a final remark, one should notice that, in a multivariate setting, one has $f_{\mathbf{Z}}=f_{\Theta} \times f_{\mathbf{U}} \times f_{\mathbf{X}_{\text {single }}}$.

As a remark and to avoid any confusion for the reader, one should notice that, despite the disaggregated inputs, only the basic variables $\mathbf{X}$ do play a role in the physical model $\mathcal{M}(\cdot)$. Thus, these disaggregated inputs are "reaggregated" within the code such that only X variables play a role in the physical behavior. In the following, one will use the notation $\mathbf{Z}$ to deal with input variables, but the reader should be aware that only the $\mathbf{X}$ variables have a real physical sense from the failure point of view.

\subsection{Extension to the bi-level input uncertainty and pick-freeze estimators}

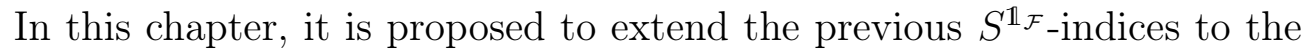
bi-level input uncertainty. To do so, the following pair of indices is proposed:

$$
\begin{aligned}
S_{i}^{\mathbb{F}_{\mathcal{F}}} & =\frac{\operatorname{Var}\left[\mathbb{E}\left[\mathbb{1}_{\mathcal{F}_{\mathbf{z}}}(\mathbf{Z}) \mid Z_{i}\right]\right]}{\operatorname{Var}\left[\mathbb{1}_{\mathcal{F}_{\mathbf{z}}}(\mathbf{Z})\right]} \\
S_{T_{i}}^{\mathbb{1}_{\mathcal{F}}} & =1-\frac{\operatorname{Var}\left[\mathbb{E}\left[\mathbb{1}_{\mathcal{F}_{\mathbf{z}}}(\mathbf{Z}) \mid \mathbf{Z}^{-i}\right]\right]}{\operatorname{Var}\left[\mathbb{1}_{\mathcal{F}_{\mathbf{z}}}(\mathbf{Z})\right]}=\frac{\mathbb{E}\left[\operatorname{Var}\left[\mathbb{1}_{\mathcal{F}_{\mathbf{z}}}(\mathbf{Z}) \mid \mathbf{Z}^{-i}\right]\right]}{\operatorname{Var}\left[\mathbb{1}_{\mathcal{F}_{\mathbf{z}}}(\mathbf{Z})\right]} .
\end{aligned}
$$

Pick-freeze estimators can be used to estimate these indices by MC simulations. In the following, these estimators will be used to get the reference results.

Computing $S_{i}^{\mathbb{1}_{\mathcal{F}}}$ and $S_{T_{i}}^{\mathbb{1}_{\mathcal{F}}}$ under a bi-level uncertainty using a single-loop CMC sampling can be achieved in four steps. The procedure presented herebelow is adapted from the one proposed by [19]. 


\subsubsection{Step \#1}

Generate $2 N$ copies of the augmented vector $\left\{\mathbf{Z}^{(j)}\right\}_{j=1}^{2 N} \stackrel{\text { i.i.d. }}{\sim} f_{\mathbf{Z}}$. These samples are stored in the following two matrices:

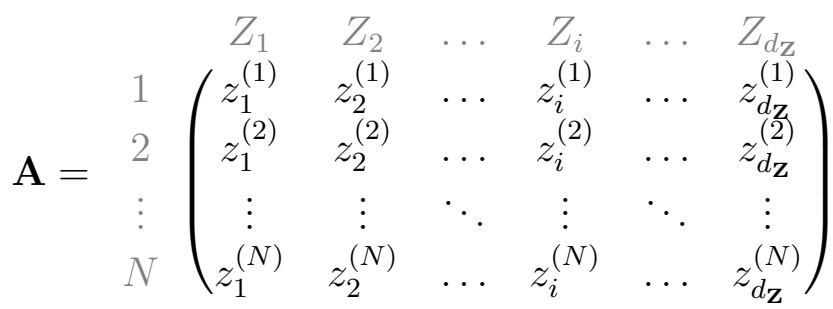

$$
\begin{aligned}
& \mathbf{B}=\begin{array}{c}
N+1 \\
N+2 \\
\vdots \\
2 N
\end{array}\left(\begin{array}{cccccc}
Z_{1} & Z_{2} & \ldots & Z_{i} & \ldots & Z_{d_{\mathbf{Z}}} \\
z_{1}^{(N+1)} & z_{2}^{(N+1)} & \ldots & z_{i}^{(N+1)} & \ldots & z_{\left.d_{\mathbf{Z}}+2\right)}^{(N+2)} \\
z_{1}^{(N+2)} & z_{2}^{(N+2)} & \ldots & z_{i}^{(N+2)} & \ldots & z_{d_{\mathbf{z}}}^{(N+2)} \\
\vdots & \vdots & \ddots & \vdots & \ddots & \vdots \\
z_{1}^{(2 N)} & z_{2}^{(2 N)} & \ldots & z_{i}^{(2 N)} & \ldots & z_{d_{\mathbf{Z}}}^{(2 N)}
\end{array}\right) .
\end{aligned}
$$

\subsubsection{Step \#2}

Generate a set of matrices $\mathbf{C}^{(i)}$, with $i \in\left\{1, \ldots, d_{\mathbf{z}}\right\}$ where the $i$-th column of $\mathbf{C}^{(i)}$ comes for $\mathbf{A}$ and all other $d_{\mathbf{Z}}-1$ columns come from $\mathbf{B}$ :

$$
\mathbf{C}^{(i)}=\left(\begin{array}{cccccc}
Z_{1} & Z_{2} & \ldots & Z_{i} & \ldots & Z_{d_{\mathbf{Z}}} \\
z_{1}^{(N+1)} & z_{2}^{(N+1)} & \ldots & z_{i}^{(1)} & \ldots & z_{\left.d_{\mathbf{Z}}+1\right)} \\
z_{1}^{(N+2)} & z_{2}^{(N+2)} & \ldots & z_{i}^{(2)} & \ldots & z_{d_{\mathbf{Z}}}^{(N+2)} \\
\vdots & \vdots & \ddots & \vdots & \ddots & \vdots \\
z_{1}^{(2 N)} & z_{2}^{(2 N)} & \ldots & z_{i}^{(N)} & \ldots & z_{d_{\mathbf{z}}}^{(2 N)}
\end{array}\right) .
$$

\subsubsection{Step \#3}

Compute the indicator function values for each sample in the matrices A, B and $\left.\mathbf{C}^{(} i\right)$. Finally, one gets the following set of $N$-dimensional column vectors:

$$
\mathbb{1}_{\mathbf{A}}=\mathbb{1}_{\mathcal{F}_{\mathbf{z}}}(\mathbf{A}), \quad \mathbb{1}_{\mathbf{B}}=\mathbb{1}_{\mathcal{F}_{\mathbf{z}}}(\mathbf{B}), \quad \mathbb{1}_{\mathbf{C}^{(i)}}=\mathbb{1}_{\mathcal{F}_{\mathbf{z}}}\left(\mathbf{C}^{(i)}\right)
$$


4.4.4. Step \#4

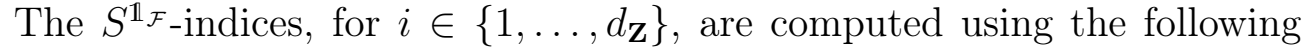
CMC estimators:

$$
\begin{aligned}
\widehat{S}_{i} \mathbb{1}_{\mathcal{F}} & =\frac{\widehat{D}_{i}-\widehat{\widetilde{P}}_{\mathrm{f}}^{2}}{\widehat{D}} \\
\widehat{S}_{T_{i}} \mathbb{1}_{\mathcal{F}} & =1-\frac{\widehat{D}_{-i}-\widehat{\widetilde{P}}_{\mathrm{f}}{ }^{2}}{\widehat{D}}
\end{aligned}
$$

where:

$$
\begin{aligned}
\widehat{\widetilde{P}}_{\mathrm{f}} & =\frac{1}{2 N} \sum_{j=1}^{N}\left(\mathbb{1}_{\mathbf{A}}^{(j)}+\mathbb{1}_{\mathbf{B}}^{(j)}\right) \\
\widehat{\widetilde{P}}_{\mathrm{f}}^{2} & =\frac{1}{N} \sum_{j=1}^{N} \mathbb{1}_{\mathbf{A}}^{(j)} \mathbb{1}_{\mathbf{B}}^{(j)} \\
\widehat{D} & =\widehat{\widetilde{P}}_{\mathrm{f}}-\widehat{\widetilde{P}}_{\mathrm{f}}^{2} \\
\widehat{D}_{i} & =\frac{1}{N} \sum_{j=1}^{N} \mathbb{1}_{\mathbf{A}}^{(j)} \mathbb{1}_{\mathbf{C}^{(i)}}^{(j)} \\
\widehat{D}_{-i} & =\frac{1}{N} \sum_{j=1}^{N} \mathbb{1}_{\mathbf{B}}^{(j)} \mathbb{1}_{\mathbf{C}^{(i)}}^{(j)}
\end{aligned}
$$

where $\mathbb{1}_{\mathbf{A}}^{(j)}, \mathbb{1}_{\mathbf{B}}^{(j)}$ and $\mathbb{1}_{\mathbf{C}^{(i)}}^{(j)}$ are, respectively, the $j$-th component of the column vector $\mathbb{1}_{\mathbf{A}}, \mathbb{1}_{\mathbf{B}}$ and $\mathbb{1}_{\mathbf{C}^{(i)} \text {. }}$.

In the following, reference values for $S^{\mathbb{1}_{\mathcal{F}}}$-indices will be estimated using this four-step procedure. Note that, following [19], an extra step could be added to compute analytical formulas to get the estimation error. However, in the following, this error is controled by use of repetitions of the whole procedure. At this point, one should notice that this single-loop CMC sampling procedure may become inefficient in the context of rare event probability estimation as most of the indicator functions in Eq. (31) could be equal to zero. Moreover, the simulation cost associated to this single-loop procedure is $N_{\text {cost }}=N\left(d_{\mathbf{z}}+2\right)$ calls to the LSF. To avoid such a computational burden, [19] proposes to use other importance sampling algorithms (in the context 
of single-level ROSA). In the next section, another way is investigated to efficiently obtain the indices under a bi-level uncertainty by an efficient estimation scheme coupling the estimators presented in Subsection 3.2, a subset sampling technique and kernel density estimation.

\section{Efficient estimation using subset sampling and kernel density estimation}

\subsection{The problem of estimating the optimal distribution at failure}

By adapting the expressions given in Eq. (18) and Eq. (19) to the bi-level uncertainty, one gets the following estimators:

$$
\begin{aligned}
& \widehat{S}_{i}{ }^{\mathbb{F}_{\mathcal{F}}}=\frac{\widehat{\widetilde{P}}_{\mathrm{f}}}{1-\widehat{\widetilde{P}}_{\mathrm{f}}} \operatorname{Var}\left[\frac{\widehat{f}_{Z_{i} \mid \mathcal{F}}\left(Z_{i}\right)}{f_{Z_{i}}\left(Z_{i}\right)}\right] \\
& \widehat{S}_{T_{i}}^{\mathbb{1}_{\mathcal{F}}}=1-\frac{\widehat{\widetilde{P}}_{\mathrm{f}}}{1-\widehat{\widetilde{P}}_{\mathrm{f}}} \operatorname{Var}\left[\frac{\widehat{f}_{\mathbf{Z}^{-i} \mid \mathcal{F}}\left(\mathbf{Z}^{-i}\right)}{f_{\mathbf{Z}^{-i}}\left(\mathbf{Z}^{-i}\right)}\right]
\end{aligned}
$$

where $\widehat{\widetilde{P}}_{\mathrm{f}}$ is the estimated value of the predictive failure probability as presented in Subsection 4.1, obtained here by ARA/SS. As suggested by these estimators adapted to the bi-level uncertainty, the first and total sensitivity indices require the estimation of $f_{Z_{i} \mid \mathcal{F}}$ and $f_{\mathbf{Z}^{-i} \mid \mathcal{F}}$. These densities correspond, respectively, to the marginal distribution w.r.t. $Z_{i}$ and to the "joint minus one" (abbreviated as "joint-1", the one corresponding to the $i$-th marginal) distribution of the failure points. The estimation of these two quantities exhibits a number of challenges that are detailed below in the dedicated paragraphs.

As a remark, one should notice that these densities $f_{Z_{i} \mid \mathcal{F}}$ and $f_{\mathbf{Z}^{-i} \mid \mathcal{F}}$ are closely related to the theoretical optimal densities required to achieve optimal variance reduction in the context of importance sampling and subset sampling [see, e.g., 30]. Indeed, such densities are known to be challenging to be estimated due to multiple reasons. Therefore, kernel density estimation (KDE) techniques are very popular to tackle this kind of issue [see, e.g., 31]. A brief review of multivariate KDE is given in the Info-box 1.

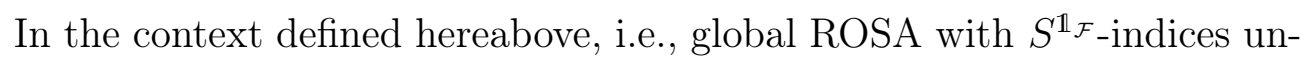
der a bi-level input uncertainty, one needs to recall the major challenges 
arising from the estimation of the optimal distribution at failure using KDE, and the new challenges induced by the use of the estimators in Eq. (39) and Eq. (40).

\subsubsection{Challenge \#1: input dimension}

It is known that the performance of $\mathrm{KDE}$ deteriorates with the input dimension. However, in the estimators presented above, this issue is predominantly affecting the total index $\widehat{S}_{T_{i}}^{\mathbb{1}_{\mathcal{F}}}$ as it requires the estimation of the joint-1 density $f_{\mathbf{Z}^{-i} \mid \mathcal{F}}$ at failure. However, this issue is intrinsically affected by the fact that, under a bi-level uncertainty, one considers the augmented

vector $\mathbf{Z}=\left(\mathbf{V}_{\text {dis }}, \mathbf{X}_{\text {single }}\right)^{\top}$ of size $d_{\mathbf{Z}}>d$ due to the disaggregated variables contained in $\mathbf{V}_{\text {dis }}$. This increased input dimension is the counterpart of taking the bi-level input uncertainty into account.

\subsubsection{Challenge \#2: complex shape of the optimal density at failure}

Another key issue arises from the fact that the shape of the optimal density at failure may be complex. By complex, one means that it is a truncated distribution (i.e., truncated by the LSS), which can be multimodal and may thus arise from a complex combination of the inputs. Note that the multimodality can be either smooth (close modes) or very sharp, especially in the context of disconnected failure regions of almost equal importance.

\subsubsection{Challenge \#3: limited number of failure points in the elite set}

In the context of rare event probability estimation, the number of failure points, i.e., samples that lie in the true failure domain, is often limited (e.g., with the CMC technique). Even if variance reduction techniques such as IS or SS can be used and may significantly increase the number of failure points in the last iteration, the elite set $\mathcal{E}$ is often of rather small cardinal compared to the total number of simulations used to get an estimate of the failure probability. Finally, one can also add more samples by using resampling within this failure domain by using, for instance, Markov Chain Monte Carlo (MCMC) sampling techniques such as the Metropolis-Hastings algorithm (or a modified version as proposed in [16]). A more detailed presentation of these algorithms is given in Chapter 6. In the context of costlyto-evaluate computer codes, resampling may even become untractable. As a result, the learning procedure of $f_{Z_{i} \mid \mathcal{F}}$ and $f_{\mathbf{Z}^{-i} \mid \mathcal{F}}$ by KDE has to be performed over a limited elite set composed of, at least, a few hundred to a few thousand failure points. 


\subsubsection{Challenge \#4: accuracy of the KDE}

A last challenge concerns the fact that, in Eq. (39) and Eq. (40), not only the estimations of $f_{Z_{i} \mid \mathcal{F}}$ and $f_{\mathbf{Z}^{-i} \mid \mathcal{F}}$ are required but also their evaluations on samples $Z_{i}$ and $\mathbf{Z}^{-i}$. Moreover, these indices are based on the variance of ratios of these density evaluations. Thus, one needs to ensure that the KDE provides sufficiently accurate estimations for the densities to avoid large estimation errors. Consequently, if a coarse KDE can be sufficient to get a sampling density able to generate samples within a specific region such as a failure region (see, e.g., the nonparametric importance sampling techniques as proposed by [32]), getting a very accurate estimation of a multivariate density over a limited set of samples is far more challenging.

If the traditional KDE formulation such as presented in the Info-box 1 may be sufficient for basic "estimation-sampling" tasks used in reliability assessment (e.g., as used inxs nonparametric importance sampling algorithms), it is definitely not efficient enough regarding the four challenges listed hereabove. To do so, one needs to focus on dedicated tools developed w.r.t. these challenges. Recently, a modified formulation of a traditional G-KDE has been proposed in [33]. This formulation is briefly reviewed below.

\subsection{Data-driven tensorized kernel density estimation}

Introduced by [33], a new data-driven tensorized G-KDE has been proposed so as to overcome a few difficulties of traditional G-KDE. For the sake of clarity and conciseness, only a few prerequisites and the core modifications are presented below. The interested reader is invited to refer to [33] for any further information.

\subsubsection{Context and prerequisites}

This data-driven tensorized G-KDE relies on the assumption that the maximum available information consists of a set of independent realizations $\{\mathbf{Z}\}_{j=1}^{N}$ of a random vector $\mathbf{Z}$. In the reliability context, such a dataset can be typically the elite set denoted by $\mathcal{E}_{\mathbf{z}}$, i.e., a $N$-dimensional vector of realizations of the input vector whose coordinates fall in the failure domain.

Another prerequisite consists in assuming that the unknown underlying distribution $f_{\mathbf{Z}}$ of these data samples might be concentrated on an unknown subset of $\mathbb{R}^{d}$. This assumption may be particularly true when the distribution of $\mathbf{Z}$ may exhibit a strong stochastic dependence structure. In the reliability context, this case typically corresponds to the case of the optimal 
density at failure for complex LSFs (even if the input vector of basic variables $\mathbf{X}$ is made of independent variables). This theoretical problem of probability concentration is strongly linked with mathematical concepts related to dimension reduction via diffusion maps theory [see, e.g., 34]. The basic idea of diffusion maps is to identify the underlying manifold upon which the data is embedded. Nonetheless, this theoretical topic is beyond the scope of this chapter and the interested reader is invited to refer to the references mentioned above for any further information.

\subsubsection{Main features of the data-driven tensorized G-KDE}

Regarding the potential constraints and challenges mentioned above, [33] propose two main modifications to the traditional G-KDE. These two features are detailed below:

- the data-driven feature: while considering a dataset $\mathcal{E}_{\mathbf{z}}=\left\{\mathbf{Z}^{(1)}, \ldots, \mathbf{Z}^{\left(N_{\text {fail }}\right)}\right\}$, the idea is to modify the traditional G-KDE estimator so that the mean and covariance matrix are equal to the empirical moments $\widehat{\mathbf{m}}_{\mathcal{E}_{\mathbf{z}}}$ and $\widehat{\Sigma}_{\mathcal{E}_{\mathbf{z}}}$ estimated from the dataset $\mathcal{E}_{\mathbf{z}}$. Moreover, it is proposed to replace the "Silverman's rule of thumb" (see the Info-box 1) characterized by the bandwidth matrix given in Eq. (44) parametrized by a single scalar bandwidth $\eta_{\text {Silv }}$ given in Eq. (45). Instead, it is proposed to use the maximum likelihood estimate $\mathbf{H}^{\mathrm{ML}}$ based on the available data;

- the tensorization feature: to take into account the possible complex dependence structure of the underlying distribution of interest, it is proposed to consider a block-by-block decomposition to separate the components of $\mathbf{Z}$ which can be reasonably assumed to be independent from those which can be assumed dependent. Thus, two dependent inputs should belong to the same block. This leads to consider a tensorized version of the bandwidth matrix $\mathbf{H}^{\mathrm{ML}}$ parametrized by a set of bandwiths $\eta_{l}$ with $l \in\left\{1, \ldots, n_{\text {block }}\right\}$.

\subsection{Methodology based on subset sampling and data-driven tensorized G- $K D E$}

The proposed methodology can be summarized as proposed in Figure 2. Thus, it can be decomposed into two major phases:

- Phase \#1: the first phase (cf. the blue blocks in Figure 2) corresponds to a reliability analysis phase. The idea is to perform a rare 
event probability estimation using ARA/SS. To do so, one needs first to set the augmented problem by constructing the augmented vector using the disaggregated strategy. Then, one performs an augmented SS (i.e., ARA/SS) which implies to set and tune several inner parameters algorithms for ARA/SS (see Chapter 6) so as to get the an estimate of the predictive failure probability and an elite set. A possible final step consists, if necessary, in resampling within the failure domain (using a dedicated MCMC algorithm as described in Chapter 6);

- Phase \#2: the second phase (cf. the orange blocks in Figure 2) corresponds to a learning and sensitivity analysis phase. To do so, one needs first to estimate the empirical moments based on the available elite set. Then, one can run the procedure of data-driven tensorized G-KDE as set in [33]. Note that, in this chapter, the algorithm used to find the block-dependence structure is a greedy algorithm. Another possibility could be to use a genetic algorithm as achieved in [33]. Finally, one can evaluate the $S^{\mathbb{1}_{\mathcal{F}}}$-indices using estimators given in Eq. (39) and Eq. (40).

In the following, this methodology is applied and tested on two academic test-cases and one realistic aerospace test-case.

\section{Application examples}

The numerical applications presented in this section are based on the following tools:

- the rare event probability estimation has been implemented in Matlab ${ }^{\circledR}$ and performed using an in-house rare event simulation toolbox developed at ONERA;

- a Python ${ }^{\mathrm{TM}}$ implementation of the data-driven tensorized G-KDE procedure proposed by [33].

The numerical testing of the methodology proposed in this chapter, as summed up in Table 1, relies on the following settings:

- firstly, the idea is to highlight the fact that dealing with bi-level uncertainty, especially with disaggregated random variables, may provide more information about the way epistemic uncertainty plays a role on 
the variability of the indicator function. To do so, one proposes a comparison of $S^{\mathbb{1}_{\mathcal{F}}}$-indices under single- and bi-level uncertainty, obtained using aboth $\mathrm{CMC}$ and $\mathrm{ARA} / \mathrm{CMC}$ with large sample sizes;

- secondly, it is proposed to efficiently estimate the bi-level $S^{\mathbb{1}_{\mathcal{F}}}$-indices using the methodology described in Subsection 5.3 (i.e., the coupling between ARA/SS and the data-driven tensorized G-KDE, abbreviated as "SS + G-KDE" in the following for the sake of conciseness), at a lower computational cost than CMC (see Subsection 4.4). To do so, one considers that several elite sets $\left\{\mathcal{E}_{\mathbf{z}}^{(j)}\right\}_{j=1}^{n_{\text {set }}}$ are obtained by repeating $n_{\text {set }}$ times the ARA/SS algorithm. Then, based on these elite sets, one can then repeat the overall procedure proposed in Subsection 5.3 so as to estimate the indices. However, due to possible numerical instabilities

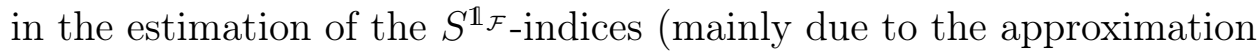
of the PDFs), the mean estimate is given together with a "success rate" which indicates the percentage of estimated values that have been kept to get the mean estimate. The rejected ones correspond to negative values of the indices which are considered as outliers but are taken into account in the score provided by the success rate.

- thirdly, after validation of the method, the impact of the increasing rareness of the failure event (regarding a limited available simulation budget) is studied as an extension of the first test-case (see the three black stars $\star$ in Table 1$)$.

Table 1: Overall strategy for the numerical tests of the proposed methodology.

\begin{tabular}{|c|c|c|c|c|}
\hline \multicolumn{2}{|l|}{ Test-case } & \multirow{2}{*}{$\begin{array}{c}\text { Single-level } \\
\text { CMC }\end{array}$} & \multicolumn{2}{|c|}{ Bi-level } \\
\hline & & & Ref. ARA/CMC & $\mathrm{ARA} / \mathrm{SS}$ \\
\hline Example $\# 1$ : Polynomial function ${ }^{a}$ & (cf. 6.1) & $\mathbf{\square} \star$ & $\boldsymbol{\square} \star$ & $\boldsymbol{a} \star$ \\
\hline Example $\# 2:$ Truss structure ${ }^{b}$ & (cf. 6.2) & 口 & $\mathbf{\square}$ & 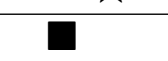 \\
\hline Example \#3: Launcher stage fallout ${ }^{c}$ & (cf. 6.3) & $\square$ & $\square$ & $\square$ \\
\hline
\end{tabular}

${ }^{a} d_{\mathbf{Z}}=5$.

${ }^{b} d_{\mathbf{Z}}=7$.

${ }^{c} 6$ basic variables, 2 uncertain parameters, $g(\cdot)$ nonlinear.

As shown in Table 1, the proposed methodology is first applied on two academic test-cases, respectively a polynomial toy-case and the failure of a 
roof structure modeled by a truss (the black squares stand for the performed calculations). Finally, a numerical application on a realistic aerospace testcase is then proposed so as to illustrate both the performances and the limits of the proposed method.

\subsection{Example \#1: a polynomial function toy-case}

\subsubsection{Description}

In a first example issued from [9], one considers an analytical toy-case made of a polynomial function whose failure is given by the following LSF:

$$
g(\mathbf{X})=y_{\mathrm{th}}-\mathcal{M}(\mathbf{X})=y_{\mathrm{th}}-\left(1+X_{1}\right)\left(5+X_{2}\right)\left(10+X_{3}\right)
$$

where the $X_{i}$, for $i=1, \ldots, d$, are three independent standard Gaussian variables. The failure is supposed to occur as soon as the output value exceeds the threshold $y_{\mathrm{th}}$. In the following, two cases are treated:

- $y_{\mathrm{th}}=250$ : in this case, the reference failure probability estimated using CMC (with $N=10^{6}$ samples and $N_{\text {rep }}=100$ replications) is $P_{\mathrm{f}, \mathrm{ref}}=8.55 \times 10^{-4}$;

- $y_{\mathrm{th}}=350$ : in this case, the rareness of the failure event is increased so as to reach a reference failure probability under a single-level uncertainty of $P_{\mathrm{f}, \text { ref }}=1.17 \times 10^{-5}$ (estimated using $\mathrm{CMC}$ with $N=10^{7}$ samples and $N_{\text {rep }}=100$ replications). For this case, the values of the standard deviation of the prior distributions are increased so as to get stronger sensitivities (see the values marked with the symbol $\star$ in Table 2).

Under a bi-level uncertainty, one considers that the probabilistic model of the first input variable $X_{1}$ is not perfectly known (see Table 2). Thus, one considers the following decomposition:

$$
X_{1}=M_{X_{1}}+S_{X_{1}} U_{X_{1}}
$$

where $M_{X_{1}}$ and $S_{X_{1}}$ follow respectively some prior distributions described in Table 2. As for $U_{X_{1}}$, it represents the natural variability of the input $X_{1}$. 
Table 2: Input probabilistic model for Example \#1.

\begin{tabular}{llll}
\hline Variable $X_{i}$ & Distribution & Mean $\mu_{X_{i}}$ & S.d. $\sigma_{X_{i}}$ \\
\hline$X_{1}$ & Normal & $\mu_{X_{1}}$ uncertain $^{a}$ & $\sigma_{X_{1}}$ uncertain \\
$X_{2}$ & Normal & 0 & 1 \\
$X_{3}$ & Normal & 0 & 1 \\
\hline$M_{X_{1}}$ & Normal & 0 & $0.1 / 0.7(\star)$ \\
$S_{X_{1}}$ & Normal & 1 & $0.1 / 0.7(\star)$ \\
$U_{X_{1}}$ & Normal & 0 & 1 \\
\hline
\end{tabular}

\footnotetext{
${ }^{a}$ For fixed values $\mu_{X_{1}}=0$ and $\sigma_{X_{1}}=1$, $P_{\mathrm{f}, \mathrm{ref}}=8.55 \times 10^{-4}$.
}

\subsubsection{Results}

Figure 3a and Figure 3b provide the reference results for the estimation of both first-order and total $S^{\mathbb{1}_{\mathcal{F}}}$-indices under a single-level uncertainty. Based on these plots, one can notice firstly that $X_{1}$ is the most influential variable on the indicator function, and second, that all the three variables show high values for the total indices which indicates that these variables present strong interactions at failure (which is a particular feature of the $S^{\mathbb{1}_{\mathcal{F}}}$-indices already pointed out by [5, Chap. 1]).

Now, one can compare these results with the reference ones obtained under a bi-level uncertainty using the ARA/CMC pick-freeze estimators presented in Subsection 4.4. The most influential input variable $X_{1}$ has been disaggregated. Thus, Figure $4 \mathrm{a}$ and Figure $4 \mathrm{~b}$ show that it is $U_{X_{1}}$ which is the most influential regarding first-order indices. Then, looking at total indices, one can notice that, even if the ranking is still preserved $\left(U_{X_{1}}>X_{2}>X_{3}\right)$, $S_{X_{1}}$ is most influential than $M_{X_{1}}$ and is of the same order of magnitude as $X_{3}$. Consequently, considering an extra level of uncertainty plays, not only a role on the reliability assessment (here, the failure probability slighty increases from $8.55 \times 10^{-4}$ to $1.0 \times 10^{-3}$ ), but also on the relative influence of the random variables at failure. Moreover, these results show that epistemic uncertainty affecting distribution parameters might play a non-negligible role compared to aleatory uncertainty of the basic variables.

Numerical results obtained by the proposed methodology are given in Table 3. These results have been obtained for the following settings: $n_{\text {set }}=10$ elite sets of $N_{\text {fail }}=2.5 \times 10^{3}$ failure samples have been obtained by repetition of the ARA/SS algorithm. In addition to that, one generates $N_{\text {gen }}=5 \times 10^{4}$ 
samples on which one evaluates the densities and compute the variance of the ratio. Thus, one can notice, by comparing the second and third columns, that the first order indices are rather correctly estimated, at a relative moderate cost and with a high success rate. One can still note that the index associated to $U_{X_{1}}$ is a little bit under-estimated. As for the total indices, even if the orders of magnitude are almost correct, most of them are a little bit overestimated.

Table 3: Results for Example \#1.

\begin{tabular}{lllllll}
\hline Variable & \multicolumn{3}{c}{ First order indices } & \multicolumn{3}{c}{ Total indices } \\
& Ref. CMC & SS + G-KDE & Success rate & Ref. CMC & SS + G-KDE & Success rate \\
\hline$M_{X_{1}}$ & $1.4 \times 10^{-4}$ & $8.4 \times 10^{-5}$ & $(100 \%)$ & 0.145 & 0.224 & $(90 \%)$ \\
$S_{X_{1}}$ & $4 \times 10^{-4}$ & $5.8 \times 10^{-4}$ & $(100 \%)$ & 0.366 & 0.476 & $(100 \%)$ \\
$U_{X_{1}}$ & 0.118 & 0.069 & $(100 \%)$ & 0.987 & 0.981 & $(100 \%)$ \\
$X_{2}$ & $5.1 \times 10^{-3}$ & $4.1 \times 10^{-3}$ & $(100 \%)$ & 0.772 & 0.806 & $(100 \%)$ \\
$X_{3}$ & $1.4 \times 10^{-3}$ & $1.5 \times 10^{-3}$ & $(100 \%)$ & 0.487 & 0.604 & $(100 \%)$ \\
\hline
\end{tabular}

Considering the influence of the failure event rareness (i.e., for $y_{\text {th }}=350$ ), numerical results are given in Table 4 . For this case, the reference predictive failure probability is such that $\widetilde{P}_{\mathrm{f}, \mathrm{ref}}=2.05 \times 10^{-5}$. Reference values for $S^{\mathbb{1}_{\mathcal{F}}}$-indices have been obtained by ARA/CMC, with $N=10^{8}$ samples and $N_{\text {rep }}=100$ replications. Numerical results for the proposed methodology have been obtained for the following settings: $n_{\text {set }}=10$ elite sets of $N_{\text {fail }}=$ 500 failure samples have been obtained by repetition of the SS algorithm (with $5 \times 10^{3}$ samples/step, an $\alpha_{\mathrm{SS}}$-quantile set to 0.75 and a final MCMCbased resampling step of $5 \times 10^{3}$ samples whose only the first $10 \%$ samples are kept). In addition to that, one generates $N_{\text {gen }}=5 \times 10^{5}$ samples on which one evaluates the densities and compute the variance of the ratio. Thus, one can notice that the first order indices are rather correctly estimated, at a relative moderate cost compared to $\mathrm{CMC}$ and high success rates. As for the total indices, almost all of them have been correctly estimated. However, the smallest one, associated to $M_{X_{1}}$, is over-estimated at a low success rate of $50 \%$. 
Table 4: Results for Example \#1 $(\star)$.

\begin{tabular}{lllllll}
\hline Variable & \multicolumn{3}{c}{ First order indices } & \multicolumn{3}{c}{ Total indices } \\
& Ref. CMC & SS + G-KDE & Success rate & Ref. CMC & SS + G-KDE & Success rate \\
\hline$M_{X_{1}}$ & $7.8 \times 10^{-8}$ & $2.6 \times 10^{-6}$ & $(100 \%)$ & 0.176 & 0.394 & $(50 \%)$ \\
$S_{X_{1}}$ & $2.0 \times 10^{-5}$ & $3.5 \times 10^{-5}$ & $(100 \%)$ & 0.539 & 0.517 & $(100 \%)$ \\
$U_{X_{1}}$ & 0.026 & 0.016 & $(100 \%)$ & 0.998 & 0.990 & $(100 \%)$ \\
$X_{2}$ & $4.6 \times 10^{-4}$ & $1.3 \times 10^{-3}$ & $(100 \%)$ & 0.902 & 0.906 & $(100 \%)$ \\
$X_{3}$ & $7.4 \times 10^{-5}$ & $8.7 \times 10^{-5}$ & $(100 \%)$ & 0.659 & 0.700 & $(100 \%)$ \\
\hline
\end{tabular}

\subsection{Example \#2: a truss structure}

\subsubsection{Description}

The second example (issued from [19]) is a roof structure whose behavior is modeled by a truss as sketched in Figure 5. For the bars, two different materials are assumed: the top boom and the compression bars are reinforced by concrete (denoted by the subscript " $c$ " in the mechanical characteristics) while the bottom boom and the tension bars are made of steel (denoted by the subscript " $s$ " in the mechanical characteristics). A uniformly distributed load $q$ is applied to the roof. As a result of the modeling, one can reduce it to nodal loads $P=q l / 4$ applied respectively on nodes $C, D$ and $F$. The failure of such a structure can be attained if the deflection of node $C$ (denoted by $\Delta_{C}$ ) reaches a given threshold $\Delta_{\text {th }}$. Such a scenario is given by the following LSF:

$$
g(\mathbf{X})=\Delta_{\text {th }}-\mathcal{M}(\mathbf{X})=0.025-\Delta_{C}
$$

The analytical formulation of $\Delta_{C}$ can be derived from the basic principles of structural mechanics applied to trusses. As a result, one has the following deflection formula [19]:

$$
\Delta_{C}=\frac{q l^{2}}{2}\left(\frac{3.81}{A_{c} E_{c}}+\frac{1.13}{A_{s} E_{s}}\right)
$$

where $l$ denotes the total length of the basis of the truss, $A_{c}$ and $A_{s}$ the sectional areas of, respectively, the concrete and steel bars, and finally $E_{c}$ and $E_{s}$ the Young's modulus of concrete and steel. Thus, one has to consider a 6-dimensional input random vector, such that $\mathbf{X}=\left(q, l, A_{s}, A_{c}, E_{s}, E_{c}\right)^{\top}$, composed of independent Gaussian variables as described in Table 5. 
Considering a single-level of uncertainty in input, the reference failure probability estimated using CMC (with $N=10^{6}$ samples and $N_{\text {rep }}=100$ replications) is $P_{\mathrm{f}, \text { ref }}=1.27 \times 10^{-2}$. Under a bi-level uncertainty, one assumes that the probabilistic model of the first input variable $X_{1}$ is not perfectly known (see Table 2). Thus, one considers the following decomposition:

$$
X_{1}=M_{X_{1}}+\sigma_{X_{1}} U_{X_{1}}
$$

where $M_{X_{1}}$ follow respectively a prior distribution described in Table 5, while $\sigma_{X_{1}}$ is supposed to be well known. As for $U_{X_{1}}$, it represents the natural variability of the input $X_{1}$ and is thus modeled by a standard Gaussian random variable.

Table 5: Input probabilistic model for Example \#2.

\begin{tabular}{llll}
\hline Variable $X_{i}$ & Distribution & Mean $\mu_{X_{i}}$ & S.d. $\sigma_{X_{i}}$ \\
\hline$X_{1}=q\left(\mathrm{~N} \cdot \mathrm{m}^{-1}\right)$ & Normal & $\mu_{X_{1}}$ uncertain $^{a}$ & $0.08 \times 20000$ \\
$X_{2}=l(\mathrm{~m})$ & Normal & $\mu_{X_{2}}=12$ & $0.02 \times \mu_{X_{2}}$ \\
$X_{3}=A_{s}\left(\mathrm{~m}^{2}\right)$ & Normal & $\mu_{X_{3}}=9.82 \times 10^{-4}$ & $0.06 \times \mu_{X_{3}}$ \\
$X_{4}=A_{c}\left(\mathrm{~m}^{2}\right)$ & Normal & $\mu_{X_{4}}=0.04$ & $0.2 \times \mu_{X_{4}}$ \\
$\left.X_{5}=E_{s}\left(\mathrm{~N}^{-2}\right)^{-2}\right)$ & Normal & $\mu_{X_{5}}=1.2 \times 10^{11}$ & $0.07 \times \mu_{X_{5}}$ \\
$X_{6}=E_{c}\left(\mathrm{~N} \cdot \mathrm{m}^{-2}\right)$ & Normal & $\mu_{X_{6}}=3 \times 10^{10}$ & $0.08 \times \mu_{X_{6}}$ \\
\hline$M_{X_{1}}$ & Normal & 20000 & $0.05 \times 20000$ \\
$U_{X_{1}}$ & Normal & 0 & 1 \\
\hline
\end{tabular}

${ }^{a}$ For the fixed value $\mu_{X_{1}}=20000, P_{\mathrm{f}, \text { ref }}=1.27 \times 10^{-2}$.

\subsubsection{Results}

Figure 6a and Figure 6b provide the reference results for the estimation of both first-order and total $S^{1_{\mathcal{F}}}$-indices under a single-level uncertainty. Based on these plots, one can notice first, that $X_{4}$ presents the largest first order index, and second, that the total indices indicate a partition into three groups of inputs: $\left(X_{4}, X_{1}\right)$ show the strongest indices, then $\left(X_{2}, X_{3}, X_{5}\right)$ have quasisimilar values for their indices, and finally $X_{6}$ (these results are coherent with those obtained by [19]).

Under a bi-level uncertainty, it is assumed that the mean of $X_{1}$ is affected by epistemic uncertainty. Thus, $X_{1}$ has been desaggregated into $M_{X_{1}}$ and $U_{X_{1}}$. As a result, Figure $7 \mathrm{a}$ and Figure $7 \mathrm{~b}$ show that it is $U_{X_{1}}$ is the 
second most influential input regarding first-order indices. As for total indices, one can see that the group $\left(X_{4}, U_{X_{1}}\right)$ is the most influential, followed by $\left(M_{X_{1}}, X_{2}, X_{3}, X_{5}\right)$ and finally $X_{6}$. Thus, again, the epistemic uncertainty affecting the mean of $X_{1}$ plays a non-negligible role on the variability of the indicator function, mainly due to interactions with other inputs.

The numerical results obtained by the proposed methodology are given in Table 6. These results have been obtained for the following settings: $n_{\text {set }}=50$ elite sets of $N_{\text {fail }}=2 \times 10^{3}$ failure samples have been obtained by repetition of the ARA/SS algorithm. In addition to that, one generates $N_{\text {gen }}=1 \times 10^{4}$ samples on which one evaluates the densities and compute the variance of the ratio. Thus, one can notice, by comparing the second and third columns, that the first order indices are almost perfectly estimated (only the strongest index associated to $X_{4}$ is moderately over-estimated), at a lower cost compared to $\mathrm{ARA} / \mathrm{CMC}$ and with the highest success rates for all the indices. As for the total indices, some of them are perfectly estimated while others show a relative bias. Moreover, the ranking is slighty modified between $M_{X_{1}}$ and $X_{3}$. However, the reference values are also very close. Thus, the proposed methodology is able to catch, at least, accurate orders of magnitude. Finally, one can see that the success rates of total indices do not reach values under $82 \%$.

Table 6: Results for Example \#2.

\begin{tabular}{lllllll}
\hline Variable & \multicolumn{3}{c}{ First order indices } & \multicolumn{3}{c}{ Total indices } \\
& Ref. CMC & SS + G-KDE & Success rate & Ref. CMC & SS + G-KDE & Success rate \\
\hline$M_{X_{1}}$ & 0.012 & 0.012 & $(100 \%)$ & 0.420 & 0.346 & $(98 \%)$ \\
$U_{X_{1}}$ & 0.042 & 0.046 & $(100 \%)$ & 0.639 & 0.567 & $(100 \%)$ \\
$X_{2}$ & $8.8 \times 10^{-3}$ & $9.1 \times 10^{-3}$ & $(100 \%)$ & 0.374 & 0.297 & $(94 \%)$ \\
$X_{3}$ & 0.013 & 0.015 & $(100 \%)$ & 0.410 & 0.365 & $(94 \%)$ \\
$X_{4}$ & 0.114 & 0.145 & $(100 \%)$ & 0.672 & 0.643 & $(100 \%)$ \\
$X_{5}$ & 0.021 & 0.023 & $(100 \%)$ & 0.478 & 0.444 & $(100 \%)$ \\
$X_{6}$ & $3.2 \times 10^{-3}$ & $3.4 \times 10^{-3}$ & $(100 \%)$ & 0.229 & 0.207 & $(82 \%)$ \\
\hline
\end{tabular}

6.3. Example \#3: application to a launch vehicle stage fallback zone estimation

\subsubsection{Description}

The role of a launch vehicle (a.k.a. space launcher) is to carry a payload (e.g., a satellite) from the Earth's surface to a given orbit. A traditional expendable space launcher is composed of multiple stages, equipped with their 
propulsion systems. During the flight, uncertainties can affect several variables in multiple disciplines (e.g., on the perturbation during trajectory or propellant combustion). For instance, focusing on the optimal trajectory assessment leads to consider the separation point as a key point in terms of uncertainty analysis (see, e.g., [35] for an illustration). In practice, separation altitudes may be lower than the Kármán line (which denotes the boundary between the Earth's atmosphere and outer space at around $100 \mathrm{~km} \mathrm{high).}$ Therefore, wind perturbations might affect the system dynamics. As a consequence, the handling of uncertainties (e.g., arising from dynamic perturbations, error measurements due to sensors or varying unburned propellant left mass) plays a crucial role in the comprehension and prediction of the global system behavior. That is the reason why it is of prime importance to take it into account during the reliability analysis and the prediction of the fallout zone. A misestimation can have dramatic consequences in terms of launcher safety, human security and environmental impact.

The goal of this chapter is to present a simplified, but representative, fallout trajectory simulation model. Indeed, this model is representative of the phenomena encountered but with a reduced simulation cost (e.g., use of mass point model) while remaining challenging enough regarding the use of advanced rare event estimation techniques and sensitivity analysis methods. For more realistic problems, one can refer to [36, 37]. In these studies, other parameters are considered and investigated concerning the dynamics of the vehicle (e.g., perturbation of atmospheric density, winds). In the present chapter, they are not taken into account for the sake of simplicity and interpretation of the given results, but they remain parameters that should be incorporated in the model to get a full high-fidelity simulation model.

The launcher stage fallout simulation computer code may be represented as an input-output black-box model. The input variables are, among others, some characteristics of the launcher and some conditions (initial or arising during the flight) of the fallout phase. These inputs are affected by uncertainties and are gathered in a random vector with a given PDF. It is assumed that this PDF is described by a parametric model of density. The output corresponds to the position of fallout and is also a random variable because of the input randomness. A typical safety measure can be the probability that a stage (e.g., the first stage) falls at a distance greater than a given safety limit. Indeed this estimation is strategic for the qualification of such vehicles.

The simulation model used in this chapter can be considered as a black- 
box model denoted by $\mathcal{M}: \mathbb{R}^{d=6} \rightarrow \mathbb{R}$. Here, it is a simplified trajectory simulation code of the dynamic fallout phase of a generic launcher first stage. The avantage of a black-box model is to enlarge the applicability of the proposed statistical approaches illustrated in this chapter to any test-cases in this range of models. As a matter of fact, the following methods proposed in this chapter are said to be "non-intrusive" w.r.t. the model under study. The $d$-dimensional (here $d=6$ ) input vector of the simulation code, denoted by $\mathbf{X}$, contains the following basic variables (i.e., physical variables) modeling some initial conditions, environmental variables and launch vehicle characteristics:

$X_{1}$ : stage altitude perturbation at separation $\Delta a(\mathrm{~m})$;

$X_{2}$ : velocity perturbation at separation $\Delta v\left(\mathrm{~m} . \mathrm{s}^{-1}\right)$;

$X_{3}$ : flight path angle perturbation at separation $\Delta \gamma(\mathrm{rad})$;

$X_{4}$ : azimuth angle perturbation at separation $\Delta \psi(\mathrm{rad})$;

$X_{5}$ : propellant mass residual perturbation at separation $\Delta m(\mathrm{~kg})$;

$X_{6}$ : drag force error perturbation $\Delta C_{d}$ (dimensionless).

These variables are assumed to be independent for the sake of simplicity. As an output, the code will give back the scalar distance $Y=\mathcal{M}(\mathbf{X})$ which represents the distance $D_{\text {code }}$ between the theoretical fallout position into the ocean and the estimated one due to the uncertainty propagation.

In the context of the launch vehicle fallout case, the input variables are known to be affected by uncertainties (e.g., , due to the natural variability of wind or due to lack-of-knowledge). Thus, applying UQ methodology leads to consider a probabilistic model for the input vector $\mathbf{X}$, i.e., by assuming the existence of a joint PDF $f_{\mathbf{X}}: \mathcal{D}_{\mathbf{X}} \subseteq \mathbb{R}^{d} \rightarrow \mathbb{R}_{+}$. Since the input variables are assumed to be independent, this joint PDF corresponds to the product of the marginal PDFs $f_{X_{i}}$ of the input variables $X_{i}, i \in\{1, \ldots, d\}$. The input probabilistic model for the launch vehicle fallout case is given in Table 7 . Note that the numerical values used in this test-case are hypothetic and should not be used for industrial applications. 
Table 7: Input probabilistic model.

\begin{tabular}{llll} 
Variable $X_{i}{ }^{a}$ & Distribution & Mean $\mu_{X_{i}}$ & S.d. $\sigma_{X_{i}}$ \\
\hline$X_{1}=\Delta a(\mathrm{~m})$ & Normal & 0 & 1650 \\
$X_{2}=\Delta v\left({\left.\mathrm{~m} . \mathrm{s}^{-1}\right)}^{-}\right.$ & Normal & 0 & 3.7 \\
$X_{3}=\Delta \gamma(\mathrm{rad})$ & Normal & 0 & 0.001 \\
$X_{4}=\Delta \psi(\mathrm{rad})$ & Normal & 0 & 0.0018 \\
$X_{5}=\Delta m(\mathrm{~kg})$ & Normal & 0 & 70 \\
$X_{6}=\Delta C_{d}(1)$ & Normal & 0 & 0.1 \\
\hline
\end{tabular}

${ }^{a}$ The input variables are independent.

The input probabilistic model is given in Table 7. The code output is the distance $D_{\text {code }}$ between the theoretical fallback position into the ocean and the estimated one. For the sake of clarity, one recalls that the LSF $g(\cdot)$ can be written as follows:

$$
g(\mathbf{X})=d_{\text {safe }}-\mathcal{M}(\mathbf{X})=d_{\text {safe }}-D_{\text {code }}
$$

for which the rareness of the failure event depends on the safety threshold distance $d_{\text {safe }}$.

Table 8: Input probabilistic model under bi-level input uncertainty.

\begin{tabular}{llll}
\hline Variable $^{a}$ & Distribution & Mean & S.d. \\
\hline$X_{1}=\Delta h(\mathrm{~m})$ & Normal & $\mu_{X_{1}}=0$ & $\sigma_{X_{1}}=1650$ \\
$X_{2}=\Delta v\left(\mathrm{~m} . \mathrm{s}^{-1}\right)$ & Normal & $\mu_{X_{2}}$ uncertain $^{b}$ & $\sigma_{X_{2}}=3.7$ \\
$X_{3}=\Delta \gamma(\mathrm{rad})$ & Normal & $\mu_{X_{3}}$ uncertain & $\sigma_{X_{3}}=0.001$ \\
$X_{4}=\Delta \psi(\mathrm{rad})$ & Normal & $\mu_{X_{4}}=0$ & $\sigma_{X_{4}}=0.0018$ \\
$X_{5}=\Delta m(\mathrm{~kg})$ & Normal & $\mu_{X_{5}}=0$ & $\sigma_{X_{5}}=70$ \\
$X_{6}=\Delta C_{d}(1)$ & Normal & $\mu_{X_{6}}=0$ & $\sigma_{X_{6}}=0.1$ \\
$\Theta_{2}=\mu_{X_{2}}\left(\mathrm{~m} . \mathrm{s}^{-1}\right)$ & Normal & $\xi_{1}=\mu_{\mu_{X_{2}}}=0$ & $\xi_{2}=\sigma_{\mu_{X_{2}}}=3.7$ \\
$\Theta_{3}=\mu_{X_{3}}(\mathrm{rad})$ & Normal & $\xi_{3}=\mu_{\mu_{X_{3}}}=0$ & $\xi_{4}=\sigma_{\mu_{X_{3}}}=0.001$ \\
\hline
\end{tabular}

${ }^{a}$ The basic variables are independent.

${ }^{b}$ For fixed values $\mu_{X_{2}}=0$ and $\mu_{X_{3}}=0$, one has:

$\triangleright$ for $d_{\mathrm{safe}}=15 \mathrm{~km}, P_{\mathrm{f}, \mathrm{ref}}=1.36 \times 10^{-4}$;

$\triangleright$ for $d_{\text {safe }}=20 \mathrm{~km}, P_{\mathrm{f}, \text { ref }}=2.31 \times 10^{-7}$. 
Due to the presence of a bi-level input uncertainty and following the methodology proposed in Subsection 4.3, it is proposed to consider the following disaggregated inputs:

$$
\begin{aligned}
& X_{2}=M_{X_{2}}+\sigma_{X_{2}} U_{X_{2}} \\
& X_{3}=M_{X_{3}}+\sigma_{X_{3}} U_{X_{3}}
\end{aligned}
$$

where $M_{X_{2}}$ and $M_{X_{3}}$ follow the same prior distributions as previously presented in Table $8, U_{X_{2}}$ and $U_{X_{3}}$ are two standard Gaussian variables, and $\sigma_{X_{2}}$ and $\sigma_{X_{3}}$ are the two standard deviations defined in Table 8. Finally, one considers the following augmented input vector:

$$
\mathbf{Z}=\left(\mathbf{V}_{\text {dis }}, \mathbf{X}_{\text {single }}\right)^{\top}=\left(X_{1}, M_{X_{2}}, U_{X_{2}}, M_{X_{3}}, U_{X_{3}}, X_{4}, X_{5}, X_{6}\right)^{\top}
$$

For the numerical experiments, simulation settings have been defined as follows. Firstly, concerning the LSF, two cases are treated to investigate the influence of the rareness of the failure event:

- in the first place, the safety threshold distance is set to $d_{\text {safe }}=11 \mathrm{~km}$ (associated to a reference failure probability under single-level input uncertainty such that $P_{\mathrm{f}, \text { ref }}=6.10 \times 10^{-3}$ ). This case is used to obtain reference results (using CMC) in order to have a better insight of what is at stake in terms of sensitivity indices.

- then, the safety threshold distance is set to $d_{\text {safe }}=15 \mathrm{~km}$. In such a case, only reference results in bi-level are provided and compared to those obtained from the proposed methodology.

Finally, concerning the proposed methodology, mean estimates of the sensitivity indices are provided together with a "success rate" which indicates, in percentage of the total number of repetitions, the number of experiments that have provided positive values of indices. If the index value is negative, the numerical experiment is considered as "failed" and the estimated value is removed.

\subsubsection{Results}

Figure 9a and Figure 9b provide the reference results for the estimation of both first-order and total $S^{\mathbb{1}_{\mathcal{F}}}$-indices under single-level uncertainty. Based on these plots, one can notice first, that $X_{2}$ presents the largest first order index, and second, that the total indices indicate a partition into two 
groups of inputs: $\left(X_{2}, X_{3}, X_{6}\right)$ show the strongest indices (above 0.50) while $\left(X_{1}, X_{4}, X_{5}\right)$ have rather moderate values (around 0.15$)$. Moreover, one can see that first-order indices are, for most of them, very low while total order indices are much stronger. This clearly indicates that variables interact a lot at failure but do not contribute that much on their own to the variability of the indicator function. Such a remark has been already pointed out in the work of [5].

Under bi-level uncertainty, Figure 10a shows that, for $X_{2}, U_{X_{2}}$ is more influential than $M_{X_{2}}$, while for $X_{3}$, it is $M_{X_{3}}$ which is more influential than $U_{X_{3}}$. This clearly highlights that, due to the disaggregated version of the augmented vector, one can analyze both effects from aleatory and epistemic uncertainties. Again, the first order indices are low which indicates a poor contribution of each variable to the overall variability of the indicator. As for total indices displayed in Figure 10b, one can observe the same ranking as for first-order indices. However, one can see the epistemic uncertainties affecting the mean value of $X_{2}$ and $X_{3}$ play a major role on the variability of the indicator function and have thus to be taken into account.

When considering the case where $d_{\text {safe }}$ is set to $15 \mathrm{~km}$, numerical results obtained by the proposed methodology are given in Table 9. These results have been obtained for the following settings:

- $n_{\text {set }}=35$ elite sets obtained after 35 repetitions of the SS algorithm;

- each elite set contains $N_{\text {fail }}=2 \times 10^{3}$ failure samples resampled at the end of each application of SS;

- in addition to that, one generates $N_{\text {gen }}=1 \times 10^{5}$ samples on which one evaluates the densities and compute the variance of the ratio;

- the reference CMC is obtained by $N_{\text {rep }}=10$ repetitions of $N_{\text {sim }}=10^{5}$ samples;

- the "success rate" is obtained by counting the number of negative-valued indices removed from the statistics.

Concerning the first-order indices, one can see that, most of them are correctly estimated (despite the fact they have small values). The strongest first-order indices (i.e., those associated to $M_{X_{2}}$ and $U_{X_{2}}$ ) are rather well estimated. As for the total order indices, the results show that the indice associated to $X_{1}$ can be correctly estimated but with a very low success rate. 
As for $X_{6}$, its total index is well estimated but with a moderate success rate. The group $\left(M_{X_{2}}, U_{X_{2}}, M_{X_{3}}, U_{X_{3}}\right)$ are estimated in terms of order of magnitude but not accurately. Finally, the indices associated to $\left(X_{4}, X_{5}\right)$ are completely over-estimated.

Table 9: Results for Step \#3.

\begin{tabular}{lllllll}
\hline Variable & \multicolumn{3}{c}{ First order indices } & \multicolumn{3}{c}{ Total indices } \\
& Ref. CMC & SS + G-KDE & Success rate & Ref. CMC & SS + G-KDE & Success rate \\
\hline$X_{1}$ & $2.3 \times 10^{-3}$ & $1.1 \times 10^{-4}$ & $(100 \%)$ & 0.155 & 0.135 & $(3 \%)$ \\
$M_{X_{2}}$ & 0.014 & 0.022 & $(100 \%)$ & 0.776 & 0.598 & $(66 \%)$ \\
$U_{X_{2}}$ & 0.028 & 0.038 & $(100 \%)$ & 0.792 & 0.511 & $(77 \%)$ \\
$M_{X_{3}}$ & $9.3 \times 10^{-3}$ & 0.012 & $(100 \%)$ & 0.697 & 0.523 & $(60 \%)$ \\
$U_{X_{3}}$ & $4.7 \times 10^{-3}$ & $5.6 \times 10^{-3}$ & $(100 \%)$ & 0.620 & 0.540 & $(60 \%)$ \\
$X_{4}$ & 0 & $5.3 \times 10^{-4}$ & $(100 \%)$ & 0.137 & 0.608 & $(63 \%)$ \\
$X_{5}$ & 0 & $1.2 \times 10^{-4}$ & $(100 \%)$ & 0.132 & 0.407 & $(49 \%)$ \\
$X_{6}$ & $2.3 \times 10^{-3}$ & $2.0 \times 10^{-3}$ & $(100 \%)$ & 0.524 & 0.518 & $(51 \%)$ \\
\hline
\end{tabular}

\subsection{Synthesis about numerical results and discussion}

As a synthesis, and according to the numerical results presented previously, one can highlight a few characteristics and perspectives about the proposed methodology:

- the proposed methodology allows to investigate the impact of the relative part of epistemic uncertainty and aleatory uncertainty to the variability of the indicator function (which is directly linked to the variability of the failure probability as stated in Eq. (7));

- the coupling between ARA/SS and the data-driven tensorized G-KDE seems to provide very promising results for global ROSA under a bilevel uncertainty, especially concerning the reduction in terms of computational cost compared to CMC, since this methodology only requires a post-treatment of an elite set obtained after a reliability analysis;

- the methodology seems able to handle moderate dimensions, at least, greater dimensions for which traditional KDE fails to estimate correctly multivariate densities;

- this methodology is driven by several tuning parameters which may influence drastically the results: the quality of the elite set (influenced 
by the tuning of the SS algorithm), the number $N_{\text {fail }}$ of failure samples in the elite set and the number $N_{\text {gen }}$ of generated samples to compute the variance. As for $n_{\text {set }}$, it is important to notice that, in practice, for costly-to-evaluate computer models (and rare event probability estimation), it might not be possible to get neither multiple not largesample-size elite sets.

\section{Conclusion}

In this chapter, a new methodology is proposed to estimate, at a lower computational cost than CMC, a set of $S^{\mathbb{1}_{\mathcal{F}}}$-indices, in the context of bi-level input uncertainty. This methodology relies on the combination of three main components:

- first, a disaggregated structure of the inputs that are affected by epistemic uncertainty;

- second, the use of the SS algorithm to get an estimate of the predictive failure probability and, jointly, to use the final elite set of failure samples to estimate the $S^{\mathbb{1}_{\mathcal{F}}}$-indices;

- third, a data-driven tensorized G-KDE which allows to improve the density estimation required to compute the indices.

The combination of these three tools allows to propose a methodology whose efficiency has been observed on two numerical test-cases. As for the complex aerospace test-case, very promising results have been obtained, even if several difficulties remain in the estimation accuracy of small indices. Moreover, the influence of the failure event rareness has been investigated too. However, this work only provides promising preliminary results. Indeed as mentioned in the results, some numerical experiments can lead to negative values of the

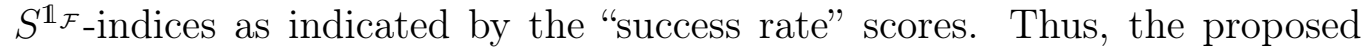
methodology might be not suited as a one-shot methodology. Its robustness should be improved, mainly by investigating the potential reasons for such a failure in terms of estimation (e.g., possible insufficiency in terms of budget allocation / inaccuracy of the KDE / inefficiency of the resampling phase using MCMC creating correlated samples). 


\section{Acknowledgements}

The first author would like to thank ONERA - The French Aerospace Lab and SIGMA Clermont as this work has been mostly achieved under a threeyear long $\mathrm{PhD}$ program co-funded by these two institutions. Moreover, the first author would like to thank several people: Émilien Flayac (PhD student at ONERA) for several valuable discussions about technical details that really helped to get this work achieved, Dr. Bertrand Iooss (EDF R\&D) and Prof. Carsten Proppe (KIT, Karlsruhe) for their advice and consideration about this work.

[1] A. Der Kiureghian and O. Ditlevsen. Aleatory or epistemic? Does it matter? Structural Safety, 31(2):105-112, 2009.

[2] E. De Rocquigny, N. Devictor, and S. Tarantola. Uncertainty in industrial practice: a guide to quantitative uncertainty management. Wiley, 2008.

[3] A. Saltelli, M. Ratto, T. Andres, F. Campolongo, J. Cariboni, D. Gatelli, M. Saisana, and S. Tarantola. Global Sensitivity Analysis. The Primer. Wiley, 2008.

[4] N. Rachdi. Apprentissage Statistique et Computer Experiments - Approche quantitative du risque et des incertitudes en modélisation. $\mathrm{PhD}$ thesis, Université Toulouse III - Paul Sabatier, 2011.

[5] P. Lemaître. Analyse de sensibilité en fiabilité des structures. PhD thesis, Université de Bordeaux, 2014. (in English).

[6] H. Raguet and A. Marrel. Target and conditional sensitivity analysis with emphasis on dependence measures. ArXiv e-prints, pages 1-48, 2018.

[7] V. Chabridon. Reliability-oriented sensitivity analysis under probabilistic model uncertainty - Application to aerospace systems. PhD thesis, Université Clermont Auvergne, 2018.

[8] J.-C. Fort, T. Klein, and N. Rachdi. New sensitivity analysis subordinated to a contrast. Communications in Statistics - Theory and Methods, 45(15):4349-4364, 2016. 
[9] G. Perrin and G. Defaux. Efficient Evaluation of Reliability-Oriented Sensitivity Indices. Journal of Scientific Computing, pages 1-23, 2019.

[10] B. Iooss and P. Lemaître. A Review on Global Sensitivity Analysis Methods. In G. Dellino and C. Meloni, editors, Uncertainty Management in Simulation-Optimization of Complex Systems: Algorithms and Applications, chapter 5, pages 101-122. Springer US, Boston, MA, 2015.

[11] P. Wei, Z. Lu, and S. Song. Variable importance analysis: a comprehensive review. Reliability Engineering and System Safety, 142:399-432, 2015.

[12] E. Zio and N. Pedroni. Monte Carlo simulation-based sensitivity analysis of the model of a thermal-hydraulic passive system. Reliability Engineering and System Safety, 107:90-106, 2012.

[13] R. B. Nelsen. An introduction to Copulas. Springer Series in Statistics. Springer-Verlag New York, Second ed. edition, 2006.

[14] R. Lebrun. Contributions à la modélisation de la dépendance stochastique. PhD thesis, Université Paris-Diderot - Paris VII, 2013. (in English).

[15] J. Morio and M. Balesdent. Estimation of Rare Event Probabilities in Complex Aerospace and Other Systems: A Practical Approach. Woodhead Publishing, Elsevier, 2015.

[16] S.-K. Au and J. L. Beck. Estimation of small failure probabilities in high dimensions by subset simulation. Probabilistic Engineering Mechanics, 16(4):263-277, 2001.

[17] S. Da Veiga. Global sensitivity analysis with dependence measures. Journal of Statistical Computation and Simulation, 85(7):1283-1305, 2015.

[18] L. Li, Z. Lu, F. Jun, and W. Bintuan. Moment-independent importance measure of basic variable and its state dependent parameter solution. Structural Safety, 38:40-47, 2012.

[19] P. Wei, Z. Lu, W. Hao, J. Feng, and B. Wang. Efficient sampling methods for global reliability sensitivity analysis. Computer Physics Communications, 183:1728-1743, 2012. 
[20] Y. Wang, S. Xiao, and Z. Lu. A new efficient simulation method based on Bayes' theorem and importance sampling Markov chain simulation to estimate the failure-probability-based global sensitivity measure. Aerospace Science and Technology, 79:364-372, 2018.

[21] A. Der Kiureghian. Analysis of structural reliability under parameter uncertainties. Probabilistic Engineering Mechanics, 23(4):351-358, 2008.

[22] A. Der Kiureghian. Measures of Structural Safety Under Imperfect States of Knowledge. Journal of Structural Engineering ASCE, 115(5):1119-1140, 1989.

[23] H. P. Hong. Evaluation of the Probability of Failure with Uncertain Distribution Parameters. Civil Engineering Systems, 13:157-168, 1996.

[24] M. Pendola. Fiabilité des structures en contexte d'incertitudes statistiques et d'écarts de modélisation. PhD thesis, Université Blaise Pascal - Clermont II, 2000. (in French).

[25] V. Chabridon, M. Balesdent, J.-M. Bourinet, J. Morio, and N. Gayton. Evaluation of failure probability under parameter epistemic uncertainty: application to aerospace system reliability assessment. Aerospace Science and Technology, 69:526-537, 2017.

[26] V. Chabridon, M. Balesdent, J.-M. Bourinet, J. Morio, and N. Gayton. Reliability-based sensitivity estimators of rare event probability in the presence of distribution parameter uncertainty. Reliability Engineering and System Safety, 178:164-178, 2018.

[27] J. C. Helton. Uncertainty and sensitivity analysis in the presence of stochastic and subjective uncertainty. Journal of Statistical Computation and Simulation, 57:3-76, 1997.

[28] S. Sankararaman and S. Mahadevan. Separating the contributions of variability and parameter uncertainty in probability distributions. Reliability Engineering and System Safety, 112:187-199, 2013.

[29] R. Schöbi and B. Sudret. Global sensitivity analysis in the context of imprecise probabilities (p-boxes) using sparse polynomial chaos expansions. Reliability Engineering and System Safety, 148:129-141, 2017. 
[30] V. Dubourg. Adaptive surrogate models for reliability analysis and reliability-based design optimization. PhD thesis, Université Blaise Pascal - Clermont II, 2011.

[31] B. W. Silverman. Density Estimation for Statistics and Data Analysis. Chapman and Hall, 1986.

[32] P. Zhang. Nonparametric Importance Sampling. Journal of the American Statistical Association, 91(425):1245-1253, 1996.

[33] G. Perrin, C. Soize, and N. Ouhbi. Data-driven kernel representations for sampling with an unknown block dependence structure under correlation constraints. Journal of Computational Statistics and Data Analysis, 119:139-154, 2018.

[34] R. R. Coifman, S. Lafon, A. B. Lee, M. Maggioni, B. Nadler, F. Warner, and S. W. Zucker. Geometric Diffusions as a Tool for Harmonic Analysis and Structure Definition of Data: Diffusion Maps. Proceedings of the National Academy of Sciences of the United States of America, 102(21):7426-7431, 2015.

[35] P. Derennes, V. Chabridon, J. Morio, M. Balesdent, F. Simatos, J.-M. Bourinet, and N. Gayton. Modeling and Optimization in Space Engineering. State of the Art and New Challenges, chapter 'Nonparametric Importance Sampling Techniques for Sensitivity Analysis and Reliability Assessment of a Launcher Stage Fallout', pages 59-86. Springer International Publishing, Switzerland, 2019.

[36] A. Ronse and E. Mooij. Statistical impact prediction of decaying objects. Journal of Spacecraft and Rockets, 51(6):1797-1810, 2014.

[37] R. Hoogendoorn, E. Mooij, and J. Geul. Uncertainty propagation for statistical impact prediction of space debris. Advances in Space Research, 61:167-181, 2018. 
In the multivariate setting, one can assume that a set $\mathcal{X}=\left\{\mathbf{x}^{(1)}, \ldots, \mathbf{x}^{(N)}\right\}$ of $N$ i.i.d. observations of a $d$-dimensional random vector, drawn from an unknown parent density denoted by $f_{\mathbf{X}}$, is available. Then, one would like to estimate this unknown parent density. Its kernel density estimator is given by:

$$
\widehat{f}_{\mathbf{X}}(\mathbf{x})=\frac{\operatorname{det}(\mathbf{H})^{-1 / 2}}{N} \sum_{j=1}^{N} K_{d}\left(\mathbf{H}^{-1 / 2}\left(\mathbf{x}-\mathbf{x}^{(j)}\right)\right)
$$

where $\operatorname{det}(\cdot)$ is the determinant operator, $K_{d}(\cdot)$ is a multivariate ( $d$-dimensional) kernel and $\mathbf{H}$ is a $(d \times d)$-dimensional positive definite symmetric matrix, often named the "bandwidth matrix". Among several widely used kernels, one can mention the Gaussian one, defined such that:

$$
K_{d}(\mathbf{x})=\frac{1}{(2 \pi)^{d / 2}} \exp \left[-\frac{1}{2} \mathbf{x}^{\top} \mathbf{x}\right]
$$

Using a Gaussian kernel corresponds to the traditional "Gaussian kernel density estimation" (G-KDE). If the choice of the kernel $K_{d}(\cdot)$ may influence the performance of the estimation in some specific cases, it remains that the most influent parameter is the bandwidth matrix $\mathbf{H}$. This matrix has to be tuned so as to find an admissible trade-off between a global smoothing and capturing the peaks of the density. It can be optimized following some optimality criteria, such that the mean square integrated error (MISE) given by:

$$
\operatorname{MISE}(\mathbf{H})=\mathbb{E}\left[\int_{\mathbb{R}^{d}}\left(\widehat{f}_{\mathbf{X}}(\mathbf{x})-f_{\mathbf{X}}(\mathbf{x})\right)^{2} \mathrm{~d} \mathbf{x}\right] .
$$

In common engineering practice (and when $d$ increases), the Gaussian assumption on $f_{\mathbf{X}}$ is often considered. Thus, a widely used approximation for $\mathbf{H}$ is derived from the so-called "Silverman's rule of thumb" [31] which assumes that $\mathbf{H}$ takes the following form:

$$
\mathbf{H}=\eta_{\text {Silv }}^{2}\left[\begin{array}{cccc}
\widehat{\sigma}_{1}^{2} & 0 & \ldots & 0 \\
0 & \widehat{\sigma}_{2}^{2} & \ddots & \vdots \\
\vdots & \ddots & \ddots & 0 \\
0 & \ldots & 0 & \widehat{\sigma}_{d}^{2}
\end{array}\right]
$$

where $\widehat{\sigma}_{i}^{2}$ for $i=1, \ldots, d$ is the empirical estimation of the variance of $X_{i}$ and $\eta_{\text {Silv }}$ is a scalar factor given by:

$$
\eta_{\text {Silv }}=\left(\frac{1}{N} \frac{4}{(d+2)}\right)^{\frac{1}{d+4}} .
$$

42

This factor only depends on the input dimension and the number of available samples.

Info-box 1: Basics of multivariate kernel density estimation. 


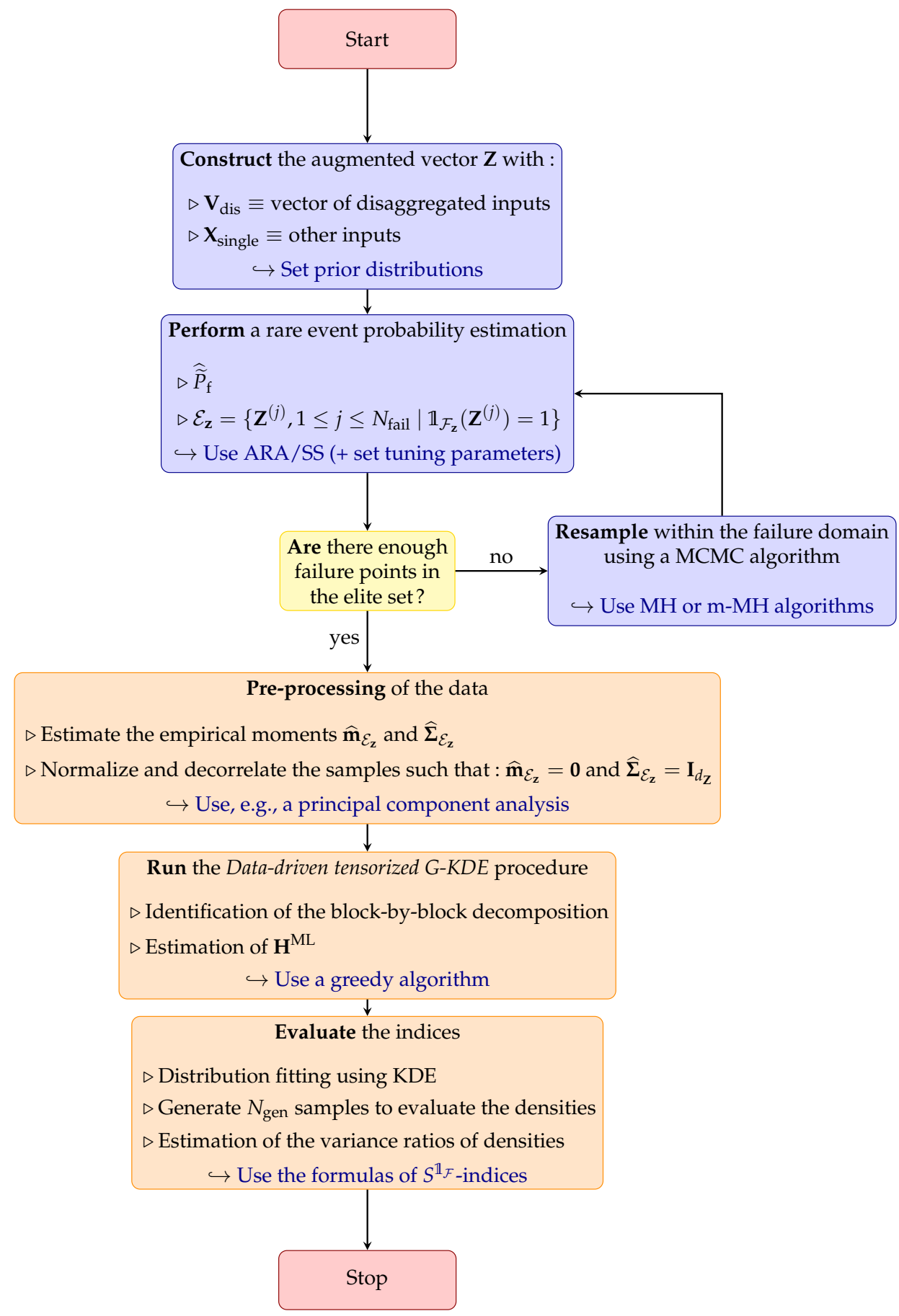

Figure 2: Flowchart of the proposed methodology to compute $S^{\mathbb{1}_{\mathcal{F}}}$-indices under a bi-level uncertainty. 


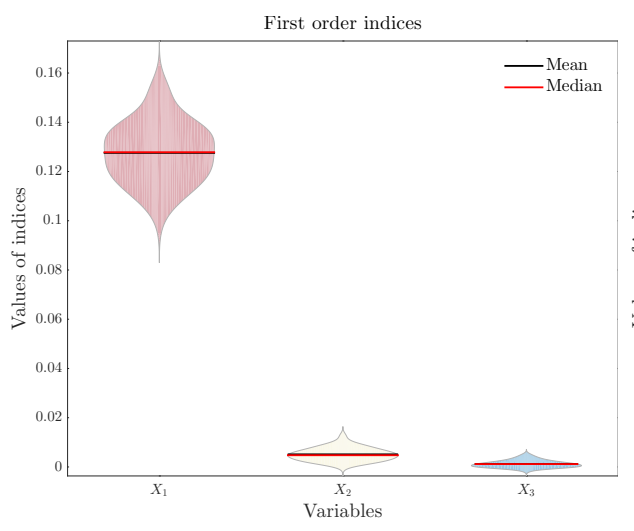

(a)

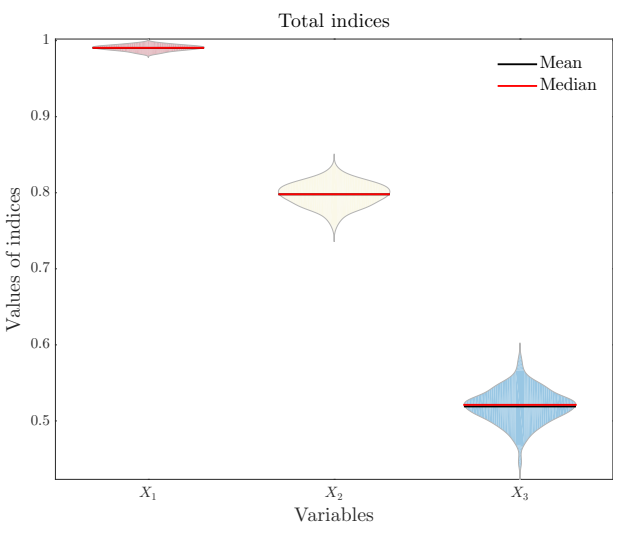

(b)

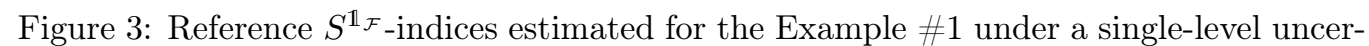
tainty $\left(\mathrm{CMC}\right.$ of $N_{\mathrm{sim}}=10^{6}$ samples and $N_{\text {rep }}=100$ repetitions, with $\left.p_{\mathrm{f}, \text { ref }}=8.55 \times 10^{-4}\right)$.

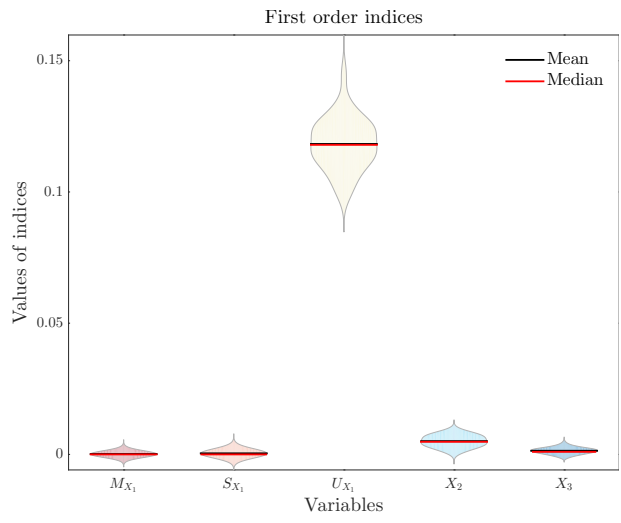

(a)

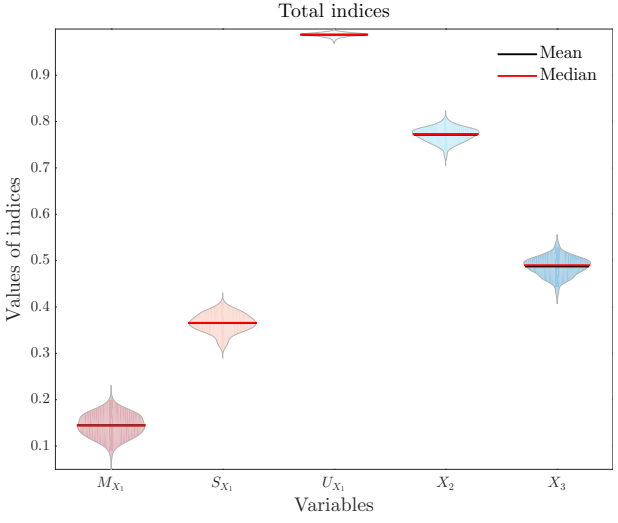

(b)

Figure 4: Reference $S^{\mathbb{1}_{\mathcal{F}}}$-indices estimated for the Example $\# 1$ under a bi-level uncertainty $\left(\mathrm{CMC}\right.$ of $N_{\mathrm{sim}}=10^{6}$ samples and $N_{\text {rep }}=100$ repetitions, with $\widetilde{P}_{\mathrm{f}, \text { ref }}=1.0 \times 10^{-3}$ ). 


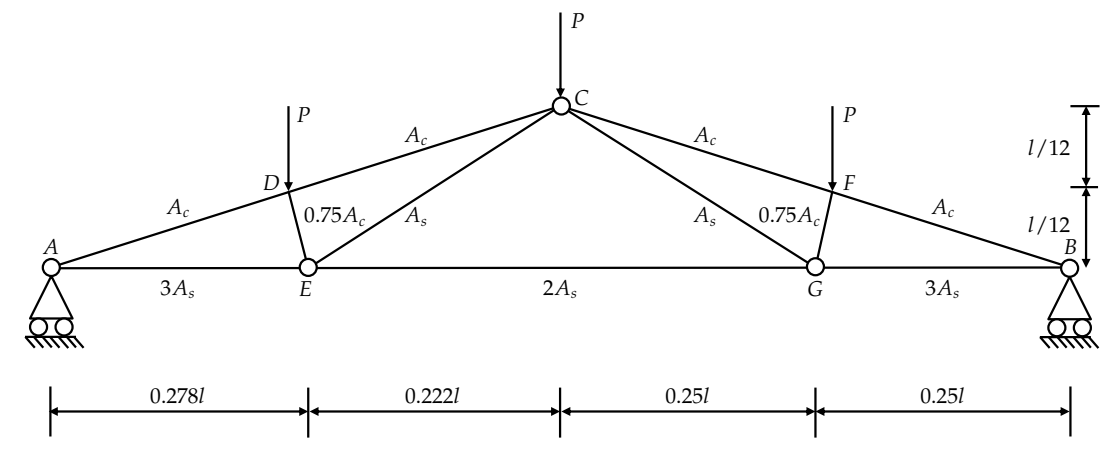

Figure 5: A roof truss.

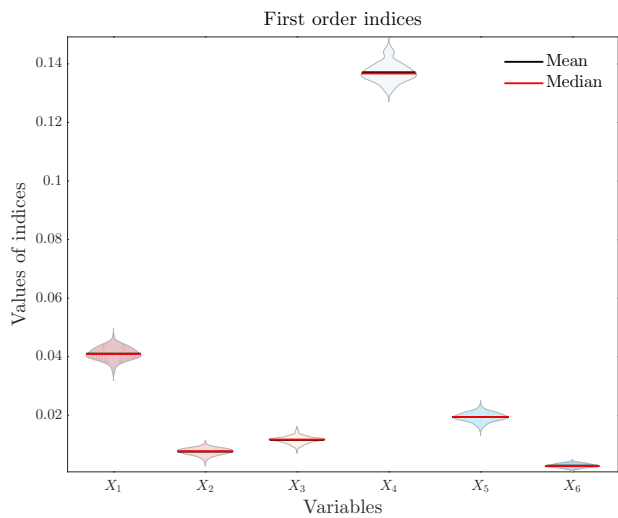

(a)

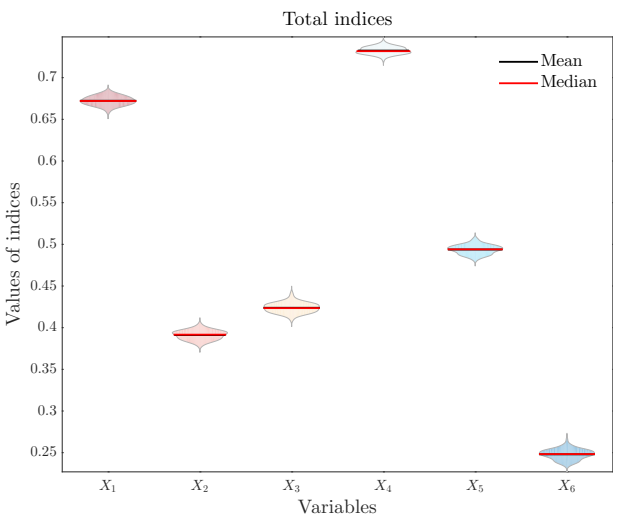

(b)

Figure 6: Reference $S^{1_{\mathcal{F}}}$-indices estimated for the Example \#2 under a single-level uncertainty $\left(\mathrm{CMC}\right.$ of $N_{\mathrm{sim}}=10^{6}$ samples and $N_{\text {rep }}=100$ repetitions, with $\left.P_{\mathrm{f}, \text { ref }}=1.26 \times 10^{-2}\right)$. 


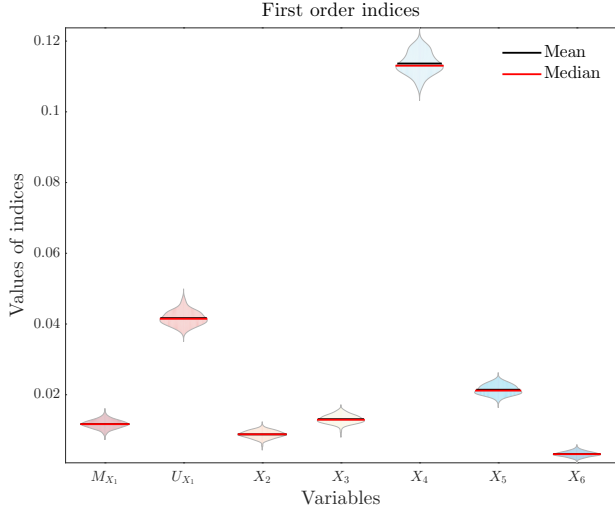

(a)

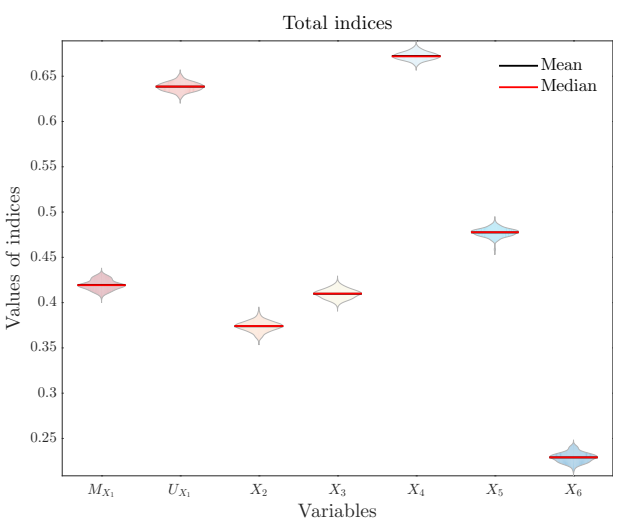

(b)

Figure 7: Reference $S^{\mathbb{1}_{\mathcal{F}}}$-indices estimated for the Example \#2 under a bi-level uncertainty $\left(\mathrm{CMC}\right.$ of $N_{\text {sim }}=10^{6}$ samples and $N_{\text {rep }}=100$ repetitions, with $\widetilde{P}_{\mathrm{f}, \mathrm{ref}}=1.65 \times 10^{-2}$ ). 


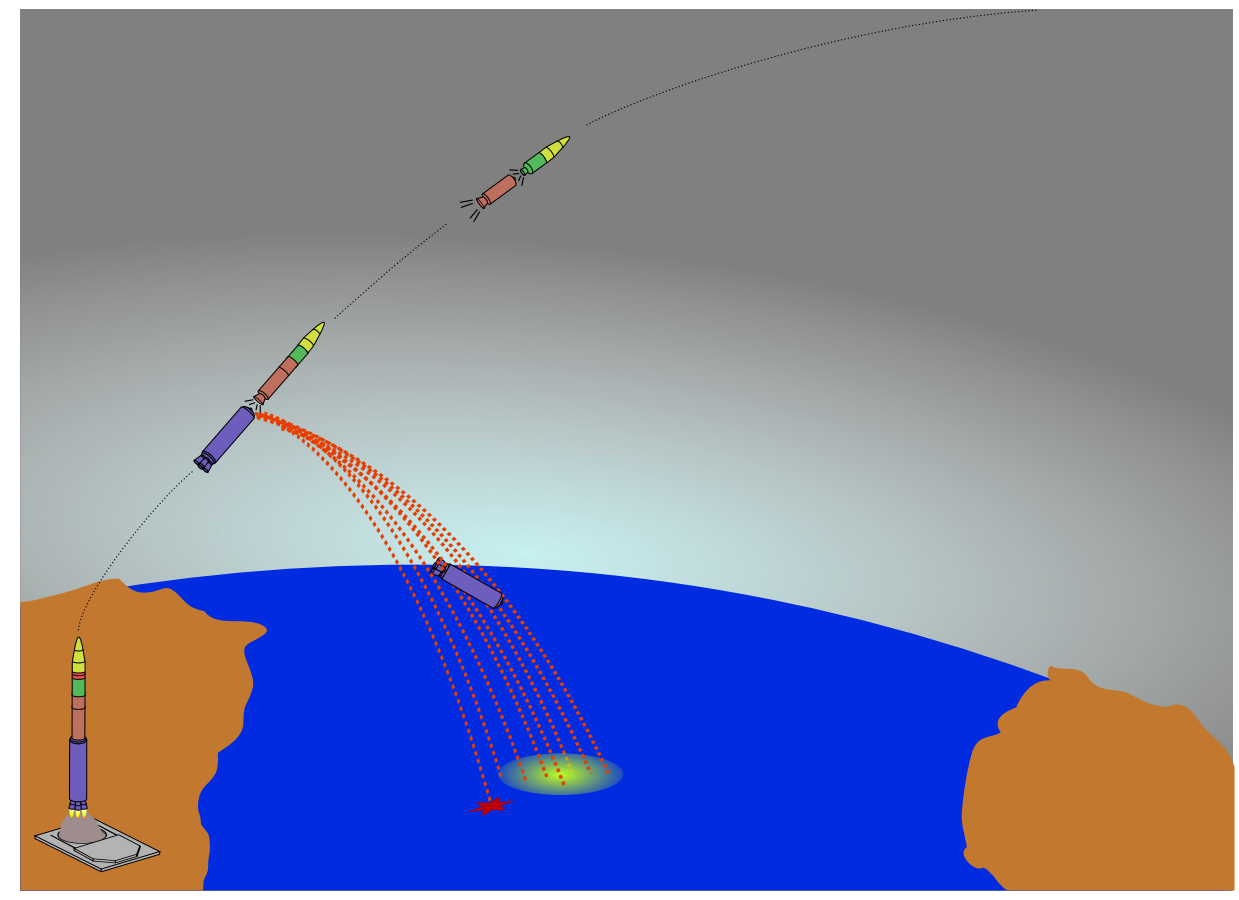

Figure 8: Illustration scheme of a launch vehicle first stage fallout phase into the Atlantic Ocean. Multiple fallout trajectories are drawn (red dotted lines), leading to the impact zone (yellow circular surface). Due to uncertainties, one fallout trajectory may lead to a failure impact point (red star). 


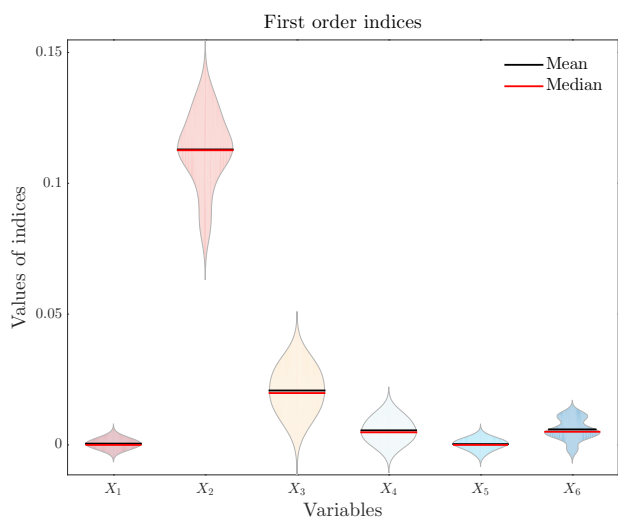

(a)

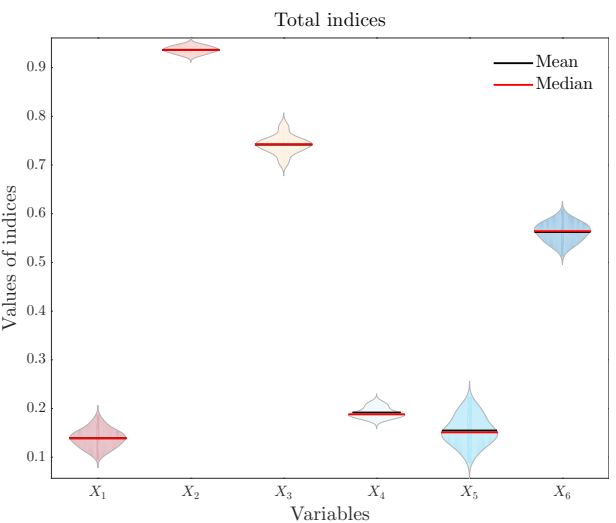

(b)

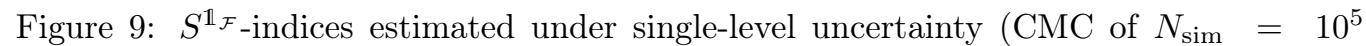
samples and $N_{\text {rep }}=10$ repetitions, with $P_{\mathrm{f}, \text { ref }}=6.10 \times 10^{-3}$ ).

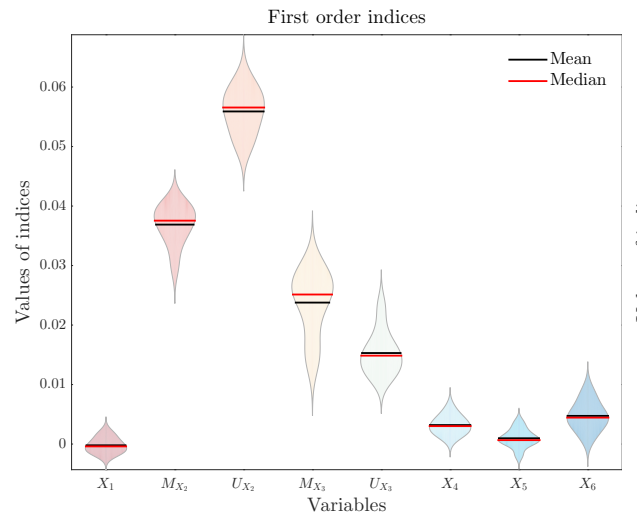

(a)

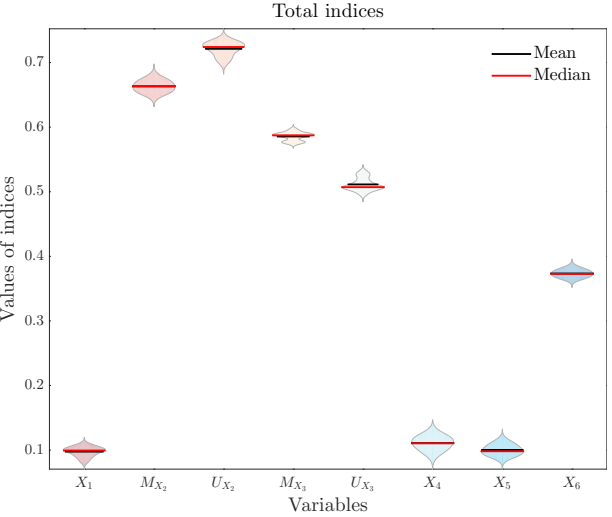

(b)

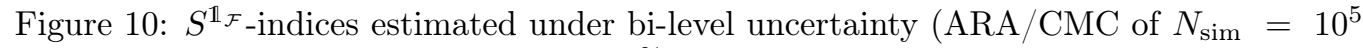
samples and $N_{\text {rep }}=10$ repetitions, with $\left.\widetilde{P}_{\mathrm{f}, \text { ref }}=3.98 \times 10^{-2}\right)$. 